ITC 2/49

Information Technology and Control

Vol. 49 / No. 2 / 2020

pp. 348-37ry

DOI 10.5755/j01.itc.49.2.24952
New Product Information Diffusion in Firm-Hosted Online Communities Based on User Influence

\begin{tabular}{|c|c|}
\hline Received 2019/12/22 & Accepted after revision 2020/05/11 \\
\hline
\end{tabular}

HOW TO CITE: Zhang, J., Guo, W., Zhao, N., Wang, J., Wang, L., Liang, R. (2020). New Product Information Diffusion in Firm-Hosted Online Communities Based on User Influence. Information Technology and Control, 49(2), 348-377. https://doi.org/10.5755/j01. itc.49.2.24952

\title{
New Product Information Diffusion in Firm-Hosted Online Communities Based on User Influence
}

\section{Jing Zhang}

Tianjin Key Laboratory of Equipment Design and Manufacturing Technology, Tianjin University, Tianjin 300072, Tianjin, China;

Department of Mechanical Engineering, Tianjin University Ren'ai College, Tianjin 301636, China; e-mail:jingzhang@tju.edu.cn

\section{Wei Guo}

Tianjin Key Laboratory of Equipment Design and Manufacturing Technology, Tianjin University, Tianjin 300072, Tianjin, China; e-mail:wguo@tju.edu.cn

\section{Nan Zhao}

School of Mechanical Engineering, Tianjin University of Technology and Education, Tianjin 300222, China; e-mail: nanzhao1982@163.com

\section{Jinliang Wang}

Tianjin Key Laboratory of Equipment Design and Manufacturing Technology, Tianjin University, Tianjin 300072, Tianjin, China; e-mail: gavinwong@tju.edu.cn

\section{Lei Wang}

Tianjin Key Laboratory of Equipment Design and Manufacturing Technology, Tianjin University, Tianjin 300072, Tianjin, China; e-mail: tjuwl@tju.edu.cn

\section{Ruoyu Liang}

School of Design, Jiangnan University, Wuxi 214122, China; e-mail: Iryasa@tju.edu.cn

\section{Corresponding author:wguo@tju.edu.cn}


The diffusion of new product information in firm-hosted online communities depends on the information interaction between users. Especially in the early stage of new product online sales, user influence based on interactive behavior is the critical factor that affects the wide and sustainable diffusion of new product information, makes users respond to the firm's announcement of new product releases in time, and improves the new product competitiveness. Since very little work has been done on the effect of user influence on the new product information diffusion, this paper proposes a model based on user influence for the information diffusion of new products in firm-hosted online communities to fill this gap. Moreover, focusing on the hot topics of new product innovation functions, we present a new method to measure user influence from the perspective of user interaction, which provides a quantitative theoretical basis for the diffusion probability of influencing user state change in new product information diffusion. Finally, our model tests a well-known firm-hosted online community in China. The results reveal that the model can effectively describe the dynamic evolution of information diffusion, explain the key dynamic factors, and accurately predict the peak time of information diffusion.

KEYWORDS: New product, user influence, information diffusion, firm-hosted online community, social networks.

\section{Introduction}

In recent years, the progress of information and communication technology has contributed to the flourishing of online social networks. The online social network has become a standard medium for people to diffuse information, share knowledge and express opinions, and the information diffusion of social networks has shown an enormous surge. Therefore, the information diffusion dynamics of social media based on the online network has become one of the hot topics. Considering that social networks have the characteristics of diversified information diffusion modes, effective diffusion rate and fission growth of users, more and more firms have realized the substantial commercial potential brought by information diffusion in online social networks, and created their own brand community platforms (i.e., firm-hosted online communities) for different purposes. For instance, IBM, LEGO, Microsoft, Toyota, and others have established online communities to gather information resources through hunger marketing, word-of-mouth communication, fan effect, to obtain user needs, mine product defects, and guide product improvement and innovation. Besides, firms may acquire external knowledge such as user preferences through information interaction with users, to better grasp the market trend and regulate public opinion. However, as far as we know, little research has been done on the dynamic diffusion mechanism of new product information in firm-hosted online communities. Hence, our study attempted to explore the information diffusion of new products from the perspective of firm-hosted online communities.
As we all know, "Diffusion of Innovation" is one of the classic theories of diffusion effect. Innovation diffusion is defined as the process of diffusion among various members of the social system in a certain way over time. From the perspective of "Diffusion of Innovation," marketing and advertising activities of new products are a sustainable and gradual process. In the early stage of the new product network sale, the wide and continuous diffusion of new product information is to make more people learn and understand the new products released by the firms. It is understood as an advertising strategy for the firm to carry out new product promotion and marketing and an important tool to realize the "sustainable marketing" of new products. Therefore, in this study, "information diffusion" is not a specific issue to discuss how to advertise, but an important academic issue - "how does advertising (new product information) diffuse among people?"

Due to the differences of information diffusion among community users with different states, researchers have spent many efforts to explore the factors that affect the process and results of information diffusion [40], [11], including the research on the value of user-generated content [30], as well as the research of user contribution and information sharing motivation [68], [19], [49]. In the firm-hosted online community, information on new products is exchanged or diffused among groups or individuals by users through community behaviors such as creating new posts, commenting, sharing, or forwarding. Users 
change from original passive recipients of information to active publishers and disseminators. Therefore, it was not enough to describe the phenomenon of information diffusion to explain the way of information dissemination [5], [63], find out where information diffusion has gone, and predict the diffusion process of information in the future. In contrast to prior studies, our research believes that it is more meaningful to find out the probability of information diffusion that affects the state change among users, to explain the causes of the dynamic evolution of information diffusion.

The effect of information diffusion of new products in the firm-hosted online community depends on whether users can make an immediate and rapid response when firms release new products online. However, previous studies only focused on the role and function of user experience and cognition after purchasing and using products for product development, innovation, and adoption, brand marketing [7]. They ignored the research on the influence of community interaction on product information diffusion based on users' past cognitive experience and limited product information when new products entered the market. Hence, in this special period that users responded to new products had released by firms, we studied the dynamic changes of users' discovery, discussion, sharing, and diffusion of new product information, aiming to help firms adjust the promotion strategy of new product information at any time. We attempted to provide a theoretical method and practical basis for firms to improve the competitiveness of new products and enhance the sustainable participation of users.

Furthermore, the characteristics of network structure are also an important research issue to explain the diffusion of new product information in firm-hosted online communities. Marketing defines information exchange as the driving force of selling new products [34]. The Internet environment also makes "sustainable marketing" of new products in the firm-hosted online community easier. However, the previous literature only considered the topological structure of the online network [22], [26], [33], and ignored the influence of the behavior and content interaction characteristics of network nodes on the initial diffusion of new product information. The individual users in the firm-hosted community are regarded as nodes in the online social network, and the interaction between nodes is the edge of the connecting nodes, which constitutes a complex social network, and nodes use the connected edge to diffuse information in the network. Most research results revealed that information diffusion is related to time, network structure [32], and interaction (relationship) strength [55]. Especially for the research of user influence, the characteristics of network structure such as degree, closeness centrality, and betweenness centrality of network nodes are usually used to measure the information diffusion ability of opinion leaders in the community. Moreover, we believe that a large number of potential information contained in the social network information generated by user behavior interaction, as well as the highly topic similarity among users on new products, have the same significantly affected the exchange or diffusion of information. Accordingly, in the research field of user influence that influences information diffusion in firm-hosted online communities, we analyzed the content similarity [23], [36] of user interaction information which could further help firms to carry out the word-of-mouth marketing of new products, implement product recommendation behavior, and build user loyalty.

Therefore, to fill the research gap and better explain the dynamic information diffusion and evolution process among individual users, the study content mainly includes the following aspects: (1) We propose a measurement method of user influence for new product information diffusion based on social relationship theory and semantic analysis method, by using big data mining. (2) We use user influence to quantify the diffusion probability that affects the change of user state in the process of new product information diffusion. (3) Furthermore, we present an information diffusion model of firm-hosted communities based on the epidemic model (SIS) to explain the way of new product information diffusion, illustrate the dynamic factors, describe its evolution trend, and effectively provide a theoretical and practical basis for firms to implement the community management and marketing strategies of new products. The remainder of this paper is organized as follows. The next section discusses related work. Section 3 proposes a new method and research model. The fourth part gives the results of the experiment and some corresponding explanations. Section 5 uses the comparison method to verify and discuss the experiment results. Section 6 summarizes the full paper. 


\section{Related Work}

\subsection{Information Research in Firm-hosted Online Communities}

At present, firm-hosted online communities are divided into two categories by researchers according to the function and service type of the platform: professional product innovation communities and comprehensive product communities. The theoretical basis of the former comes from the "user toolkits for innovation" by von Hipple [52], such as the online community of Local Motors from an American Internet start-up company, and the Idealstorm community of Dell. Through the release of innovation project information related to products or services, firms can attract users with rich professional knowledge and strong skills to participate in innovation activities. To make innovation projects continue to advance and succeed, Zheng et al. [66] studied the key factors affecting the success of innovation projects based on the diffusion of innovation (DOI). They also put forward the complexity measurement model of community innovation activities according to the characteristics of innovation projects and resources. Moreover, the professional product innovation community platform encourages users to contribute and exchange technical or creative information with a certain incentive mechanism and serve the research and development of new products. At the same time, the platform has likewise become an effective tool for enterprises to manage user innovation. Represented by lead users with innovation awareness, it promotes the research and development of product innovation projects. Guo et al. [20] defined community users as the intellectual resources of innovation communities. By analyzing their social relationship attributes, they identified six different types of resources and discussed the contribution of various types of resources to the product innovation process.

Compared with the former, the comprehensive product community often has multiple functions, such as product marketing, shopping, social interaction, and entertainment, to attract users to participate in the community interaction so that users can freely exchange product experience and discuss product shortcomings. The typical representative communities are the user innovation community of Ducati, an Italian motorcycle manufacturer, and the official forum of Xiaomi, a Chinese mobile phone manufac- turer. The valuable information that users continue to contribute is essential to the success of comprehensive product communities. By analyzing the effective information content contributed by users, firm managers can quickly and accurately grasp relevant information such as product performance defects and design deficiencies, understand the development trend of user needs, assist firms in product improvement iterations, make the effective judgment of product improvement, and grasp the future market development trend. Abrahams et al. [1] took the automobile industry as an example to describe and evaluate the text mining tools, which are used to classify user-generated content, extract valuable information from user posts, and provide sound effects and competitive intelligence for firms. Through the empirical research on the contribution of key users of Xiaomi forum, Liang et al. [30] proposed a method of product defect information mining based on the topic model and association algorithm, to automatically identify product attributes information, obtain product defect and user complaint information, and support firms to make product $\mathrm{R} \& \mathrm{D}$ and management decisions.

Users' participation in the community is not only product innovation behavior, but also social behavior. Users reply, comment, and discuss with each other in the community, forming a social network of online communities based on user interaction. User-contributed information related to innovation projects or products is also generated based on interaction. Whereas a review of the extant literature reveals that both professional product innovation communities and comprehensive product communities, ignore the crucial premise of mining the valuable information generated by users' participation in community activities. That is whether the information generated by users is continuous and wide diffusion, to attract more users to better participate in the interaction and contribute more practical and innovative information to the success of products/services. Therefore, it is of great significance for the management and marketing decision of new products of firms to study the information diffusion of social networks in firm-hosted online communities and to understand and control the diffusion trend of product information.

\subsection{Information Diffusion Model in Social Network}

In the current research on the information diffusion of the online social network, the diffusion models are 
mainly divided into two categories. It includes the explanatory models of information diffusion mechanism and the predictive models of information diffusion rule. The former model includes epidemics and influence models, and the latter model divides into the independent cascade model, graph-based linear threshold model, and game theory model. The explanatory model mainly focuses on the study of the characteristics of information diffusion, including the factors that affect information diffusion [29], the relationship between information diffusion and social networks. This kind of research helps both researchers and practitioners better understand the diffusion process of information in social networks. It also provides a basis for other extended research.

Information in social networks diffuses among different users, from the transmitter to the receiver. However, some of them refuse to receive information, some refuse to diffuse information, and some both receive and diffuse information [21]. The information diffusion process is analogous to the spread of the epidemic. Infected people infect the virus to susceptible people, and the information diffuses in the same way. That is information disseminator transmits the information to the receiver. Therefore, the epidemic model is the core of understanding the dynamic diffusion process in complex networks [41], and the epidemic model is used to explain the impact of individual characteristics of users on information diffusion. Therefore, researchers learn and use classical infectious disease models to understand the diffusion process of information, including SI (Susceptible Infected) model, SIS (Susceptible Infected Susceptible) model considering repeat infection, SIR (Susceptible Infected Removed) model, SIRS (Susceptible Removed Susceptible) model in the heterogeneous network. In recent years, different researchers have continuously improved and innovated the classical model to study the process of information diffusion more accurately. Most of the scholars focused on the classification of user state types of epidemic models in different research objects. For instance, Xiong et al. [62] added the user state C (contacted) who had read information but not diffused in the study of information diffusion of the online microblog. They put forward the SCIR (Susceptible, Contacted, Infected, and Refractory) model, believing that I and $\mathrm{R}$ are stable states of information diffusion. Wang et al. [53] attempted to describe the process of information transmission with a dynamic evolution equation and established an SEIR (Susceptible Exposed Removed) model. By adding the Exposed (E) nodes to the model, they found that the user login frequency promoted the speed and range of information transmission. Based on the SIR model, Cannarella and Spechler [4] proposed an irSIR model with adding an infection recovery dynamics process to simulate the adoption and abandonment behavior of OSN users. According to Feng et al. [12], the infection probability of a node was proportional to its fraction of infected neighbors, and the FSIR (fractional SIR) model was proposed to simulate the information diffusion process. Furthermore, to find out the factors that affect information diffusion more comprehensively, Xu et al. [64] established the S-SEIR model on SNS. They proved that information diffusion is determined by user behavior but also influenced by the value of the information itself. Wang et al. [54] introduced the emotional information as weight in the process of information diffusion between individuals; proposed ESIS (emotion-based spreader-ignorant-stifler) model, and found that information diffusion is related to the spreading probability and retweeting strength.

Indeed, the factors that affect users' state change are always associated with the infection rate and recovery rate in the model in the information diffusion process. According to the epidemic model [41], when the diffusion probability of the infected individuals to the susceptible individuals is greater than a certain critical value, the infected individuals diffuse the information until the diffusion process makes the whole network infected individuals in a stable state. Therefore, we believe that in the field of information diffusion, it is more important to study the probability of information diffusion that leads to the change of user state, quantify the user's ability to diffuse information or willingness to receive information with the rate of infection and recovery and explain the information diffusion process in essence. Nevertheless, few scholars have discussed this issue.

\subsection{User Influence in Social Networks}

In the field of information diffusion, the research of individual influence based on network interaction [51], [45] has attracted more and more scholars' attention, and it has become one of the key issues in the research of information diffusion in the social network. Based on the influence study, the research- 
ers constructed the relevant model [26], [61], which was used to find the most influential user nodes or the collection of nodes in the social network, to study the information diffusion pattern. In the previous literature, most scholars measured the importance of nodes based on the characteristics of network topology [33], [36], [35]. They used the centrality of graph theory and network analysis to measure the structural characteristics of individuals in the network and used it as an indicator to judge the importance of network nodes, to quantify the influence of individual information diffusion behavior, to find the most critical node in the network [17], and to explore the way of information dissemination. Generally speaking, the node with a higher centrality value has a greater influence on other nodes in the network, and the number of users affected is also more [24]. It is worth noting that the individual differences of social network users' information diffusion through posting, commenting, reading and other behaviors - whether the nodes in the network receive or diffuse information or not, are not only affected by individual characteristics but also closely related to the state of their neighbors. That means that the measurement index describing the individual centrality [56] should not only consider the degree centrality, closeness centrality [37], and betweenness centrality of the individual node but also pay attention to the eigenvector centrality related to the neighbor node. Furthermore, the degree of closeness between neighbors in the network also plays a crucial role in evaluating the importance of nodes. According to the hypothesis of "strength of weak ties" [17], compared with the network with highly clustered node relations, the disease or information diffusion network with "long ties" has farther distance and faster speed in spreading social behaviors. Because "long ties" establish information connection between strangers and friends, the communication between information rapidly expanded into new areas of the network, thus reducing the redundancy in the diffusion process [58], [59], [44]. Therefore, Centola [6] believed that the clustered characteristics of the network significantly affected the success of social network information diffusion. Watts [57] also used the feature of the "clustering coefficient" in the study of describing the relationship between network nodes.

Besides, Porter and Donthu [42] believed that their neighbors' behaviors influence users' behavioral preferences. Divakaran [10] has further explained that if users in social networks had similar cultural backgrounds or values, the groups formed by these users would be more cohesive. Furthermore, the relationship existing in the social network is regarded as active and free information exchange and sharing between the user nodes and their neighbors. Then, the similarity between the user's preferences for information may lead them to follow similar attitudes and behavior patterns. Therefore, the similarity of information preferences for the new product among users in firm-hosted online communities affects the acceptance of information by people around them, and thus affects the effect of information diffusion. To sum up, we believe that the influence of individual users is an important reason for the diffusion of social network information. Therefore, in the field of information diffusion, this paper calculates the influence of users from the perspective of user behavior interaction and content interaction in firm-hosted online communities. Following that, we quantify the information diffusion probability of social networks from one user to other users by utilizing user influence based on the network relationship, to explain the dynamic causes of user state transformation in the epidemic model, and to study the mode of information diffusion in the network community, which to provide marketing strategies and methods for the continuous diffusion of new product information.

\section{Method and Model}

In the firm-hosted online community network, information transmission, and communication between individuals or groups of users make information diffusion among nodes in the network formed by user interaction. Therefore, in this research, the information diffusion network of firm-hosted communities is represented as $G=(V, E)$, in which $V=\left\{v_{1}, v_{2}, \ldots, v_{n}\right\}$ is the collection of individual nodes of users participating in information diffusion in the community network. And $|V|=N$, the total number of network users is $N . E$ $\subseteq V \times V$ is a set of directed edges in the network, which represents the relationship between users formed by interactive behaviors such as posting and commenting. When the community users publish a new post, other users in the community may be influenced by opinion leaders with high network centrality or similar information preferences among users to read the posts and 
make comments with a certain probability. Through encouraging them to participate in the reading and discussion of the topic content, users are likely to publish new posts around the topic content so that the topic can be widely and continuously diffuse. Inversely, some users are not interested in the new post information in the community. They did not participate in comments or posts after reading, which hindered the diffusion of post information. Therefore, this article aims at the information diffusion of hot topics of new products in the community, mining the dynamic factors that affect users' comments on the topic and information diffusion, and defining the research variables that affect information diffusion from the perspective of user's behavior interaction and content interaction characteristics.

\subsection{Definition of Research Variables in Information Diffusion Network}

\subsubsection{User Behavior Interaction Characteristic}

We used the topological structure of the information diffusion network of the firm-hosted online community to analyze the interaction characteristics of user behavior and measure the causes of user influence. Centrality reflects the importance of individual users in the network, describes the characteristics of interaction between network nodes, which is an important variable to measure the influence of network nodes, and reflects the ability and level of individual network users to spread information.

\section{Definition 1. Degree Centrality}

The degree centrality is the most direct measure of centrality in network analysis. In the field of information diffusion or infection, degree centrality is the basic description of the information diffusion ability of users. In the social network, if user $j$ comments posting user $i$, there is an edge from node $j$ to node $i$. Calculate degree centrality $\left(C_{d}\right)$ of a node $i$ :

$$
C_{d}(I)=\frac{K_{i}}{N-1}=\sum_{j \in V} \frac{k_{i j}}{N-1},
$$

where $K_{i}$ is the degree of the node, $N$ is the total number of nodes in the network, and $k_{i j}$ is the number of nodes $j$ adjacent to the node $i$.

\section{Definition 2. Closeness Centrality}

The closeness centrality is the reciprocal of the sum of the shortest path distances from one node to all other nodes. It reflects the closeness between one node and other nodes in the network and describes the influence of nodes in the network. For a node, the closer it is to other nodes, the higher its closeness centrality is, and the greater the value of the user's information diffusion is. Closeness centrality also considered being a time factor affecting the orderly information diffusion in the network. Calculate closeness centrality $\left(C_{c}\right)$ of a node $i$ :

$$
C_{C}(i)=\frac{1}{d_{i}}=\frac{N-1}{\sum_{j \neq i} d_{i j}},
$$

where $d_{i}$ is the average shortest distance of node $i$, and $i$ and $j$ are the nodes of $V$.

\section{Definition 3. Betweenness Centrality}

The betweenness centrality is the number of times a node acts as the shortest bridge between two other nodes. The higher the number of times a node acts as a mediator, the higher its betweenness centrality. Generally, the number of shortest paths through a node is the index to describe the importance of the node. The betweenness centrality describes the influence of nodes in the network and reflects the user's control of information diffusion. Calculate betweenness centrality $\left(C_{b}\right)$ of a node $i$ :

$$
C_{b}(i)=\sum_{j \neq i \neq k} \frac{g_{j k}(i)}{g_{j k}},
$$

where the total number of shortest paths between node $j$ and node $k$ is $g_{j k}$. In graph $G$, the number of shortest paths through node $i$ between the two nodes is $g_{j k}(i)$.

\section{Definition 4. Eigenvector Centrality}

Eigenvector centrality is one of the important indexes to evaluate the importance of nodes in the network, which is used to find the most influential users in the network. It not only considers the number of neighbor nodes in the network but also examines the influence of the quality of nodes on the importance. The eigenvector centrality reflects that the influence value of a node depends only on the number of neighbors of the node, but also the critical influence degree of each neighbor node. Calculate eigenvector centrality $\left(C_{e}\right)$ of a node $i$ :

$$
C_{e}(i)=\lambda^{-1} \sum_{j=1}^{N} a_{i j} e_{j},
$$

where $\lambda$ is the maximum eigenvalue of the adjacency matrix $A$ and $\mathrm{e}=\left[e_{1}, e_{2}, \ldots, e_{n}\right]^{T}$ is the eigenvector cor- 
responding to the maximum eigenvalue $\lambda$ of the adjacency matrix $A$.

\section{Definition 5. Clustering Coefficient}

The clustering coefficient is used to measure the clustering of the network, which represents the probability that two adjacent nodes of an individual node are also adjacent in the network, reflecting the degree of clustering of the network. The closeness of the relationship between the network neighbors also plays a crucial role in evaluating nodes' importance. According to the previous literature [6], in the field of information diffusion, if the nodes and their neighbors tend to connect, it is easier to form a small-scale regional network, which means the increase of clustering coefficient hinders the diffusion of information. Therefore, we believe that if the neighbor nodes of the node are more inclined to connect other nodes in the network besides the neighbor nodes, then the information diffuses rapidly in a more extensive range. Calculate clustering coefficient (CC) of a node $i$ :

$$
C C(I)=\left[\frac{2 E_{i}}{k_{i}\left(k_{i}-1\right)}\right]^{-1},
$$

where $E_{i}$ is the actual number of edges between the $k_{i}$ neighbor nodes of node $i$.

\subsubsection{User Content Interaction Characteristic}

The users of firm-hosted online communities are mainly connected through information interaction such as posts and comments. Therefore, the information content created based on user interaction is the carrier of the user's contact. As an essential resource of community networks, the information content generated by the user's historical interaction behavior plays a significant role in promoting information diffusion among users or groups and evaluating the individual influence of users. Accordingly, it is meaningful for us to analyze and measure the formation factors of user influence and explain the way of information diffusion from the perspective of user content interaction characteristics.

\section{Definition 6. Content Similarity}

Content similarity refers to the similarity between the user's preference for new product information and subject content. The similarity between the product cognition and experience published by users in the past and the content in the new product topic is a factor that cannot be ignored to influence users to participate in the topic discussion and diffuse the new product information. We extract high-frequency vocabularies of product attributes from historical content published by users and hot topics of new products, respectively, and calculate the similarity between historical content generated by users and relevant topics with the Jaccard coefficient. The larger the Jaccard coefficient, the higher the relevance between the content published by users and the topic content, the higher the user's attention to the topic, the more significant the impact on information diffusion, and vice versa. Calculate content similarity $J(i)$ of a user $i$ :

$$
J(i)=\frac{|A \cap B|}{|A \cup B|},
$$

where $A$ is high-frequency vocabularies of product attributes from the historical content generated by user $i$, and $B$ is high-frequency vocabularies of product attributes from the contents of new product hot topics.

\section{Definition 7. Diffusion Capability}

Diffusion capability refers to the ability of community users to get the average number of other users' readings and comments before they participate in hot topics of new products and the total number of users' posts in the historic publishing behavior data to quantify the impact on information diffusion in the community. Reading posts is an effective way for users to diffuse information in the community. However, the number of posts read cannot sufficiently indicate that the users of posts have effectively diffused the relevant information on new products. Only when the users who are interested in the post comment on the post, or combine the external knowledge gained from reading the post with their subjective experience to express their views on the new product, can they fully explain that the users who post have higher diffusion ability to the new product information. Therefore, to prove whether users are productively infected with new product information in the information diffusion process, users' posts and the number of comments received should also be taken as the necessary factors to consider the information diffusion power of users. The stronger the user's diffusion capability of information content, the higher the influence of the users who publish the post. Calculate diffusion capability $D(i)$ of a user $i$ : 


$$
\begin{aligned}
D(i) & =\operatorname{Num}[\operatorname{post}(i)]+\overline{\operatorname{Num}}[\operatorname{read}(i)] \\
& +\overline{N u m}[\operatorname{comment}(i)],
\end{aligned}
$$

where $N u m[p o s t(i)]$ is the number of posts published by user $i, \overline{N u m}[\mathrm{read}(i)]$ is the average number of readings obtained by user $i$, and $\overline{N u m}[\operatorname{comment}(i)]$ is the average number of comments received by user $i$.

\subsection{Information Diffusion Model}

\subsubsection{Research Framework}

To further explain the dynamic causes of information diffusion, this paper puts forward a method of measuring user influence suitable for the firm-hosted online community of firms and constructs an information diffusion model. Based on the characteristics of network nodes of individual users and information content interaction of user's historical behavior in the communi- ty information diffusion network, the user influence is quantified from the perspective of user behavior interaction and user content interaction, respectively. This paper proposes a new product information diffusion model of the firm-hosted online community by combining user influence factors with the epidemic model. The average value of user influence in different periods is used to quantify the infection rate $\lambda$ and recovery rate $\mu$ that affect the transformation of users from the same state to another state in the model, and then the differential equation is established. The information diffusion model is purposed to find out the transition rules of different user states, describe the information diffusion process in the community network by considering the role of different user's influence on the information diffusion situation, analyze the key factors affecting the information diffusion, and predict the peak time of information diffusion.

\section{Figure 1}

Research framework

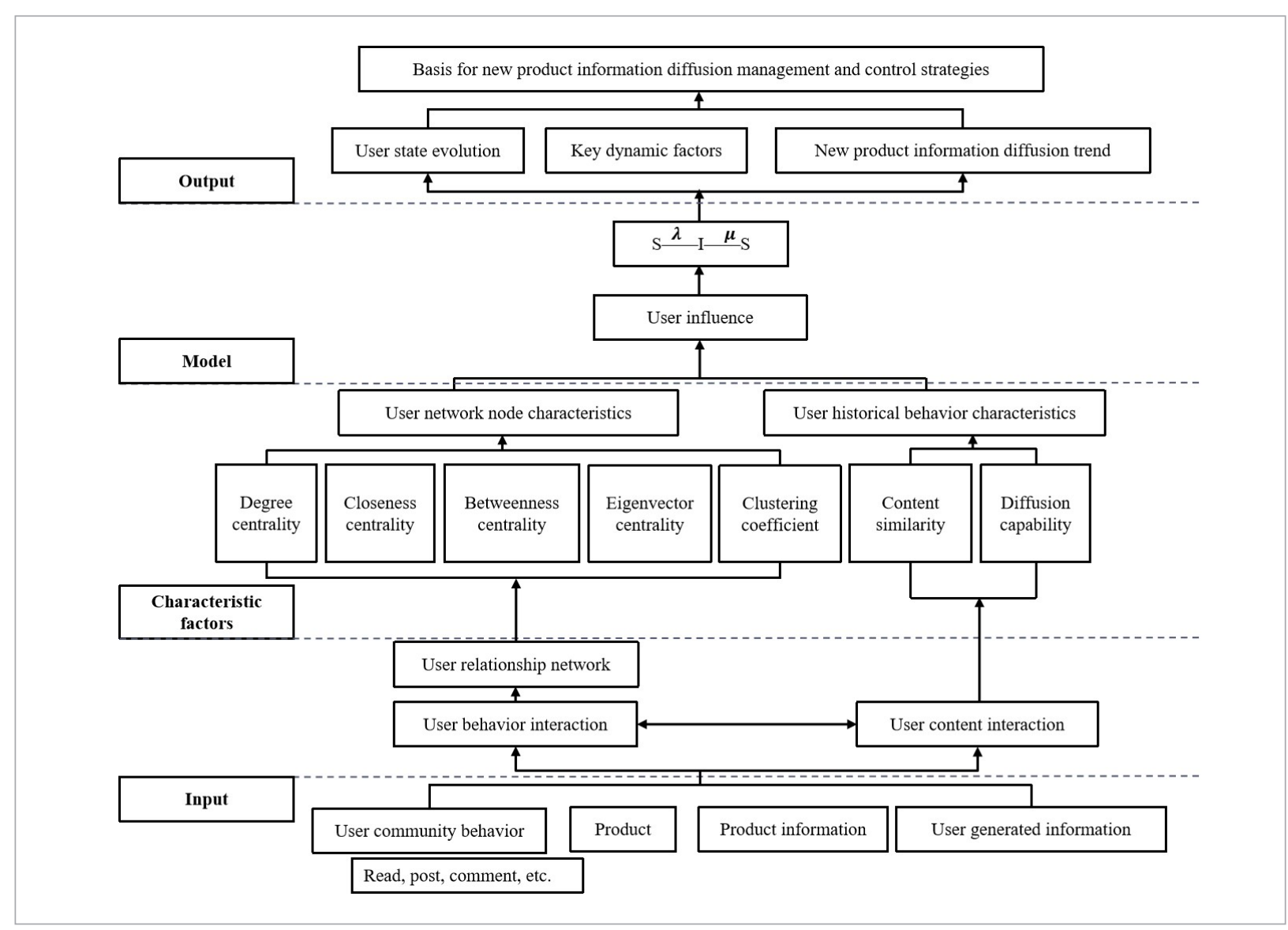




\subsubsection{User Influence}

Based on the user's interactive relation of the information diffusion network of firm-hosted online communities, this paper extracts the elements of user influence from the perspective of user behavior interaction characteristics and user content interaction characteristics to measure user influence. Considering the static network structure attributes of individual users, and the historical behavior characteristics of users, the influence function of the user $i$ is defined as:

$$
\begin{gathered}
\operatorname{Inf}(i)=\omega_{d} C_{d}(i)+\omega_{c} C_{c}(i)+\omega_{b} C_{b}(i)+\omega_{e} C_{e}(i) \\
+\omega_{c c} C_{c c}(i)+\omega_{j} J(i)+\omega_{p} D(i) \times\left(\frac{1}{2}\right)^{\frac{\Delta t}{T}},
\end{gathered}
$$

where $\omega_{d}$ is the weight coefficient of degree centrality. $\omega_{c}$ is the weight coefficient of closeness centrality. $\omega_{b}$ is the weight coefficient of betweenness centrality. $\omega_{e}$ is the weight coefficient of eigenvector centrality. $\omega_{c c}$ is the weight coefficient of the clustering coefficient. $\omega_{j}$ is the weight coefficient of content similarity. $\omega_{p}$ is the weight coefficient of diffusion capability. In this dissertation, principal component analysis (PCA) is used to determine the weight coefficient of each factor of user influence.

It is worth noting that the popularity of product posts in the community networks gradually decreases over time, so the capability of information diffusion by users weakened. To represent the life cycle of information from release to extinction, and to describe the dynamic attenuation characteristics of user information diffusion capability, we introduced the half-life function $\left(\frac{1}{2}\right)^{\frac{\Delta t}{T}}$. The half-life defined in physics refers to the time $T$ required for the radioactive atom of a radioisotope to decay to half of its original quantity. Similarly, in the field of information diffusion, we use the number of comments obtained by the most popular posts in the firm-hosted online community as the quantitative indicator of the influence of posts, and the half-life is understood as the time taken by the number of comments obtained by posts to reach half of the total number of comments. The expression is defined as:

$$
m=M\left(\frac{1}{2}\right)^{\frac{\Delta t}{T}}
$$

where $\Delta t$ is the decay time, $\Delta t=t^{\prime}-t_{0}, t^{\prime}$ is the current time, and $t_{0}$ is the initial behavior time of user $i$. Ac- cording to the real data, the half-life is set at 25 days, a total of 600 hours.

\subsubsection{New Product Information Diffusion Model of Firm-hosted Communities}

In this paper, "Susceptible-Infected-Susceptible" (SIS) epidemic model is used to describe the process of new product information diffusion in the firm-hosted online community, and construct the new product information diffusion model. SIS model is a dynamic model to study the spread of epidemic and information diffusion, which is an abstract description of the diffusion process. It is different from other epidemic models. SIS model only involves two-state variables: Susceptible (S) and Infected (I). The infected individuals do not give immunization upon recovery from infection, and individuals become susceptible again and may reach a stationary state. In a specific data set of the real social network, a user who has chosen to diffuse the information in an earlier case may no longer diffuse it or further choose to re-diffuse it after receiving the information. Hence, the information diffusion model derived from the SIS epidemic diffusion mechanism can approximate real-world scenarios of information diffusion in social networks and is widely used in the research field of complex networks [41], [37], [38]. The information diffusion model with the characteristics of social networks can describe the dynamic process of information diffusion in social networks [2], reveal the rules of information diffusion, predict the trend of information diffusion, and analyze the causes and key factors of information diffusion. Therefore, the new product information diffusion model is appropriate for our study of the firm-hosted online community.

Information diffusion is contact diffusion, which means that when the unknown information users contact the information known users, it inevitably infects with a certain probability. In the process of information diffusion, the transformation of users' individual or group states not only depends on the development of themselves but also is affected by the states of their neighbors. Therefore, the users' states of Susceptible (S) and Infected (I) in the SIS model are defined as follows. Infected individuals (I) are the people who have known information about new products in the community. They infect their neighbors by publishing new posts and comments, to achieve the purpose of diffusing new product information. Susceptible individuals 
(S) are the unknown of new product information in the community. They are easily attracted by the new product information and influenced by the infected individuals (I) to receive the information, which transformed into the infected individuals. They can choose to browse and comment to infect the susceptible neighbors with a certain probability $\beta$ of infection to continue to diffuse new product information. However, when they are no longer interested in or concerned about the information, they can also choose not to diffuse the information after receiving it, and return to susceptible with a certain recovery probability $\gamma$.

Because the diffusion of new product information in the community has the effect of explosive, short-term and high aggregation, we believe that the inflow and outflow of users participating in information diffusion in each period are equal to each other, so we do not consider the impact of factors such as birth rate and mortality. Set the total number of users as a constant $N$, the proportion of susceptible individuals and infected individuals in the total number of users at time tis $S(t)$ and $I(t)$ respectively, and $S(t)+I(t)=1$. The model is established by:

$N[I(t+\Delta t)-I(t)]=\beta N S(t) I(t) \Delta t-\gamma N I(t) \Delta t$

$N \frac{d I}{d t}=\beta N S I-\gamma N I$.

The dynamic equation of information diffusion is given by:

$$
\left\{\begin{array}{l}
\frac{d I}{d t}=\beta I(1-I)-\gamma I \\
I(0)=I_{0}
\end{array}\right.
$$

In the firm-hosted online community, the process of infection is unidirectional. Therefore, when the new product information released by infected individuals diffuses among users, the user's state changes from susceptible to infected and then returns to susceptible. As mentioned above, user influence is the decisive factor of whether susceptible individuals are infected by infected individuals to diffuse the information. Therefore, this study quantifies the infection rate and recovery rate that affect the individuals' state transition by user influence, expressed as the probability of information diffusion. In the community diffusion network, a specific state user $i$ is connected to $n$ neighbors, where node $i$ influences the state change of $k$ neighbors.
Since $k$ neighbor nodes only have the two states of Infected and Susceptible, the probability of information diffusion expressed by binomial distribution is:

$$
\begin{aligned}
& P(X=K)=C_{n}^{k} \operatorname{In} f_{t}(i)^{k}\left(1-\operatorname{In} f_{t}(i)\right)^{n-k} \\
& (k=0,1,2, \cdots, n) .
\end{aligned}
$$

Then, the information diffusion probability of any user's state transformation at time $t$ is given by:

$$
\begin{aligned}
& \beta(t)=\sum_{k=0}^{n} \frac{k}{n} C_{n}^{k} \operatorname{Inf} f_{t}(i)^{k}\left(1-\operatorname{Inf} f_{t}(i)\right)^{n-k} \\
& \quad(k=0,1,2, \cdots, n) .
\end{aligned}
$$

Therefore, the infection rate that affects the transformation of the user state in the process of information diffusion expressed as $\beta(t)$, and the recovery rate is shown as $(1-\beta(t))$. The differential equation is established and expressed as:

$$
\left\{\begin{array}{c}
\frac{d I(t)}{d t}=\overline{\beta(t)} I(1-I(t))-(1-\overline{\beta(t)}) I(t) . \\
I(0)=I_{0}
\end{array}\right.
$$

\section{Experiments}

In this part, we first give a brief description of our data set, and then present the experimental results and discuss them.

\subsection{Data Collection}

The research model was tested samples collected from an official forum of the firm-hosted online community by Xiaomi, a world-famous mobile phone manufacturer located in China. To reduce capital expenditure, risk, and develop products of commercial value, more and more companies launched their own online communities to achieve open innovation. As a mobile Internet company focused on the design and manufacture of smartphones, Xiaomi is a typical representative of the research on firm-hosted online communities [28], [46], [31].

It adopted a unified retail and ecosystem strategy and launched the official Xiaomi community forum in 2010 to make full use of opportunities across users' 24-hour digital life in emerging markets. Xiaomi forum provides an amount of product information, function discussion, and service sections to attract users to participate, such as product evaluation, discussion, and 
help, bug feedback. It also encourages users to contribute content and interact with others, motivates external experts or interested users to share and exchange experience of using products in the forum every day, and expresses their needs and expectations for the upcoming new products through comments or posts. It can assist firms grasp product relevant information to implement product innovation while understanding and controlling the diffusion trend of product information. By the first quarter of 2019, Xiaomi active users have reached hundreds of millions of people around the world [67]. As presented above, the Xiaomi forum provides a large number of data samples, which are authentic and reliable official information. Such massive information resources generated by users have the characteristics of a high degree of interaction, which is easy to form and construct the information diffusion network of the community. Therefore, the Xiaomi forum is an ideal setting for our research.

At present, Xiaomi was the fourth global smartphone vendor by market share [13]. According to the statistics of Gartner, Inc. (an authoritative market research organization), the global smartphone sales picked up in the first quarter of 2018, but not evident [16]. Nevertheless, Xiaomi's sales increased by $124 \%$ yearon-year, becoming the biggest winner. In particular, in the third quarter of 2018, Chinese brand Xiaomi helped drive global smartphone sales [14]. Moreover, as opposed to other units declining, the annual sales volume of Xiaomi reached 120 million in 2018 [15]. Xiaomi's excellent performance in 2018 largely depends on Xiaomi 8 series mobile phones. As a flagship model with Qualcomm snapdragon 845 processor and AMOLED screen, Xiaomi 8 series mobile phones quickly gained market recognition after being released. Moreover, by October 2018, the sales exceeded 6 million. Therefore, this paper makes an empirical analysis based on real data of the Xiaomi 8 series of mobile phones in the Xiaomi forum.

Xiaomi Company released a new generation of mobile phone Xiaomi 8/8se on May 31, 2018, and the performance machine Xiaomi 8 youth version defined as "invincible youth" was released on September 19, 2018. Therefore, from May 31 to September 19 is the crucial period for the diffusion of new product information in the early stage of Xiaomi 8/8se release, which is beneficial to our study of the evolution trend of new product information diffusion based on user influence and to find the critical peak time point of information diffusion. We take Xiaomi 8/8se's new product information in the forum as the research object and choose the period from May 31 to September 19 to analyze and verify the data of this information diffusion model. Next, according to the new product innovation function publicized by Xiaomi company, we determined the hot topics of new products, which are respectively "Snapdragon 845" (Topic A), "Infrared face recognition unlocking” (Topic B), "Transparent exploration version of the under-screen fingerprint" (Topic C), "The world's first dual-frequency GPS of the world" (Topic D). Through the search of hot topic keywords, new product posts, and their related comments related to innovation functions in the forum are collected and screened as research samples. Table 1 shows the statistics of four hot topics.

The effect of information diffusion of new products is not only related to users' participation behavior after product releases, but also closely related to users' historical behavior before product releases. Therefore, we collected the information of Xiaomi 6X (released on April 17, 2018), which was the previous generation of mobile phone products shared and exchanged by users, as the historical behavior data of users, to analyze the impact of historical behavior characteristics

Table 1

Statistic of four topics

\begin{tabular}{c|c|c|c|c|c}
\hline Data set & Time interval & Number of Posts & $\begin{array}{c}\text { Number of } \\
\text { Comments }\end{array}$ & $\begin{array}{c}\text { Number of } \\
\text { Posting users }\end{array}$ & $\begin{array}{c}\text { Number of Comment } \\
\text { users }\end{array}$ \\
\hline Topic A & $2018.05 .31-2018.09 .19$ & 229 & 25281 & 161 & 17854 \\
\hline Topic B & $2018.05 .31-2018.09 .19$ & 148 & 11780 & 114 & 5732 \\
\hline Topic C & $2018.05 .31-2018.09 .19$ & 565 & 19602 & 450 & 11057 \\
\hline Topic D & $2018.05 .31-2018.09 .19$ & 180 & 5700 & 141 & 3964 \\
\hline
\end{tabular}


of users on information diffusion at the early stage of new product releases.

\subsection{Data Processing}

To study the information similarity of user influence factors, we have built a high-frequency lexicon of product attributes to provide a data research basis for the information diffusion model experiment. We preprocessed the collected hot topic information data of new products in the forum. First, we deleted the posts that could not be used due to the lack of information and then removed the data noise, deleted irrelevant picture information, attachment links, and other content. The second, we summarized the dictionary of JIEBA segmentation and the professional vocabularies of Xiaomi product-related mobile phone industry, carried out word segmentation processing, marked the part of speech of products and removed the stop words, and established the vocabulary of Xiaomi 8/8se mobile phone, and obtained 3347 product attribute words in the end. Finally, we used Python to filter the high-frequency vocabularies of product attributes from the product posts of hot topics and the historical behavior data of users, and which be used to build four hot topics and the product attribute lexicon of users.

Furthermore, based on our long-term observation of user participation behavior in the Xiaomi forum, 12 hours were defined as the time segment in the information diffusion model and set it at a one-time node. According to the law of the product information life cycle, the diffusion process of Xiaomi 8/8se new prod-

\section{Table 2}

High-frequency vocabularies of product attributes (part)

\begin{tabular}{c|l}
\hline No. & \multicolumn{1}{|c}{ Product attributes words } \\
\hline 1 & 1.4um large pixel \\
\hline 2 & Qualcomm Snapdragon \\
\hline 3 & Adreno630 \\
\hline 4 & Scene identification \\
\hline 5 & 3D structured light face recognition \\
\hline 6 & AI scene mode \\
\hline 7 & 1080 p video capture \\
\hline 8 & Screen fingerprint \\
\hline 9 & Dual-frequency GPS \\
\hline 10 & RAM \\
\hline
\end{tabular}

uct information is divided into four continuous time intervals with 60-time nodes as a one-time interval. The four consecutive time intervals are expressed as time intervals I, II, III, and IV.

In the data analysis of each time interval, we found that among the users participating in the information diffusion of new products of the Xiaomi forum, some users' influence does not increase or decrease with the change of time. Through our further observations and statistics, most of the users only participate in comments and do not post, so their diffusion capability is 0 . That showed that a vast majority of users did not pay attention to the posts related to the topic of new product innovation function again after only one comment, and stopped the behavior of information diffusion. Since there was almost no multi-level comment relationship in posts of the Xiaomi forum, we only considered the interaction between posting users and commenting users. Thus, this kind of user who only had commented but never posted actively had a feeble, weak ability to infect other users. However, the number of such users would affect the probability of user state transformation and then influenced the evolution process of information diffusion. Accordingly, in the information diffusion model, we considered the effect of the cumulative number of such users on the information diffusion probability in each time interval.

Besides, we defined that in the fermentation period of new product information diffusion, that is, within 48 hours after the release of new products, users who had participated in the information interaction of various hot topics were set $I_{0}$ as the initial number of infected individuals according to the time sequence, including the posting users who did not participate in comments, and the commenting users. The latter only commented on the posts but did not post. In contrast, users who participated in posting or commenting 48 hours after the hot topic was released were considered as susceptible individuals of new product information. We believed that such users had the opportunity to be infected by the information of infected individuals, and then discussed and diffused the information on new products.

Because the research data about user influence in this paper were quite different. To eliminate the singular data, make the data index in the same order of magnitude, have comparability, suitable for comprehensive comparative evaluation. We have carried on the Gauss normalization process to the original data. 


\subsection{Data Analysis and Results}

The purpose of establishing the information diffusion model of new products is to illustrate the information diffusion process of new products in firm-hosted online communities, excavate the key driving factors affecting the transformation of different user states, study the law of information diffusion, and provide a theoretical and practical basis for firms to put forward management and marketing strategies of new products. The research approach is of considerable significance to improve the market competitiveness of new products and the sustainability of network users' awareness and attention to new product information.

\subsubsection{Analyze the Variation of the Number of Infected Individuals}

We use the information diffusion model based on the epidemic model (SIS) to analyze the rule of the number of infected individuals in the Xiaomi forum in the early stage of new product releases. The $i \sim t$ curve is defined as the curve that represents the change of the infected individuals' proportion, which shows the increasing and decreasing trend of the number of infected individuals with time. As shown in (a), (b), (c) and (d) of Figure 2, we find that the proportion of infected individuals of hot topics A, B, C and D in four-time intervals of I, II, III and IV, which increases

\section{Figure 2}

Curves of the proportion and rate of infected individuals in four-time intervals for four topics: (a), (b), (c) and (d) The changes in the proportion of infected individuals of topics A, B, C, and D in four-time intervals; (e), (f), (g) and (h) The changes in the rate of infected individuals of topics A, B, C, and D in four-time intervals

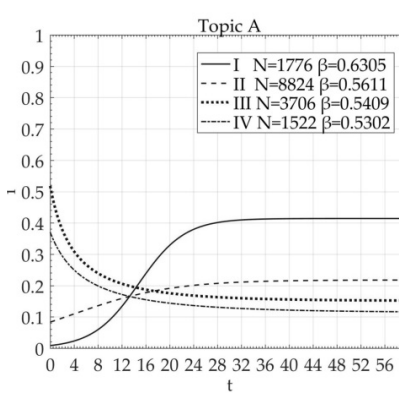

(a)

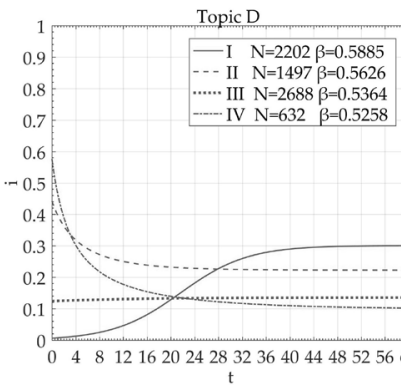

(d)

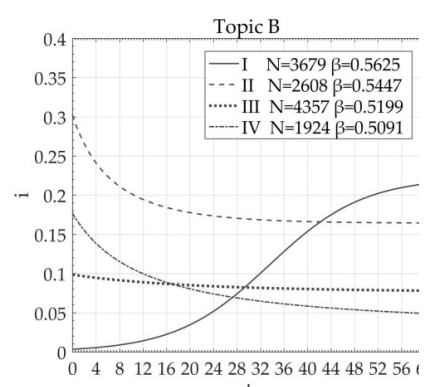

(b)

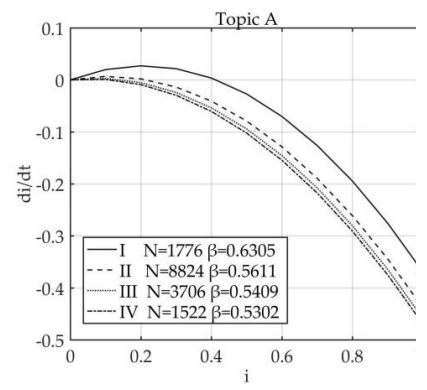

(e)

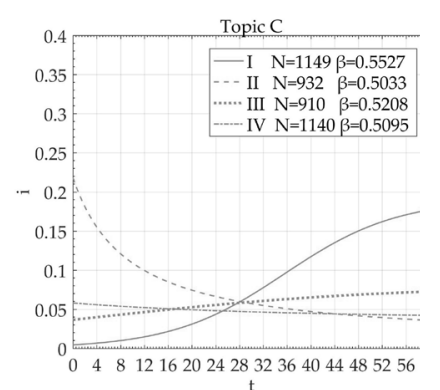

(c)

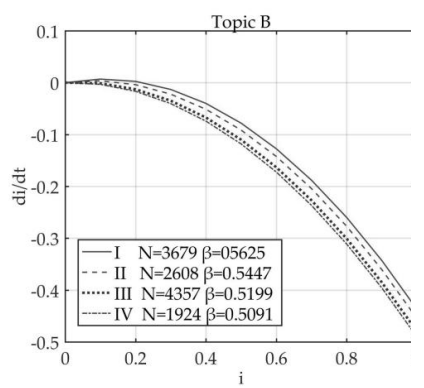

(f)

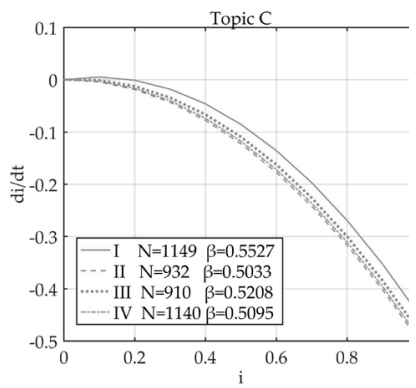

(g)

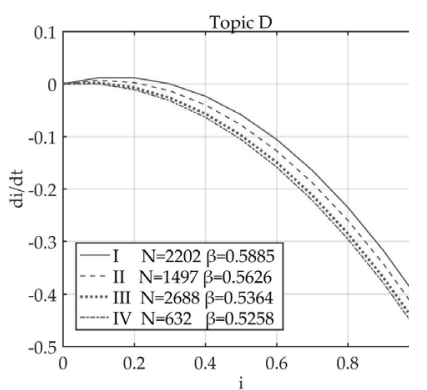

(h) 
with the increase of information diffusion probability $\beta$ and decreases with the decrease of $\beta$. Also, the $d i / d t$ curve used to represent the rate of infected individuals of four hot topics in four-time intervals. As shown in (e), (f), (g), and (h) of Figure 2, the four topics are in each time interval, the greater the probability of information diffusion, the higher the rate of infected individuals, and the smaller the information diffusion probability, the lower the rate of infected individuals. Consequently, the probability of information diffusion measured by user influence has a significant impact on the number of infected individuals and the rate of infected individuals in the process of information diffusion.

\subsubsection{Describe the Process of New Product Information Diffusion}

Figure 3(a), (b), (c), and (d) shows the proportion of infected individuals in the four-time intervals of I, II, III and IV for hot topics A, B, C, and D. The trends of change are expressed in different line styles. $\mathrm{N}$ is the total number of users participating in the topic information interaction in each time interval, which is the number of user nodes of the information diffusion network formed by user interaction in the time interval.

Through a comprehensive analysis of the experimental graphs of the four-time intervals of the information diffusion process, we can see that the proportion curves of infected individuals of Topic A and Topic B show the change of form first rising and then declining. More apparently, the number of people receiving information infection of each topic is gradually decreasing. The proportion of infected individuals in Topic $\mathrm{C}$ and Topic D presents a variation that rises first, then falls, then rises and then falls once more. That is because, in the SIS model, the number of initially infected individuals $I_{0}$ determines the trend of the proportion curve of infected individuals. Therefore, the increase or decrease of infected individuals in the process of information diffusion shown in the figure can be interpreted as the result of the influence of the initial proportion of infected individuals in each topic. Besides, when the $I_{0}$ is smaller, and the number of valid contacts per user (the number of contacts $\sigma$ is the ratio of infection rate $\beta$ to recovery rate $\gamma$ ) is higher than one during an interval of information diffusion, the $I(t)$ curve would grow in S-shape, and on the contrary, it would decline. Therefore, we be-

\section{Figure 3}

Curves of the proportion of infected individuals of topics A, B, C, and D in four-time intervals: (a) Time interval I; (b) Time interval II; (c) Time interval III; (d) Time interval IV

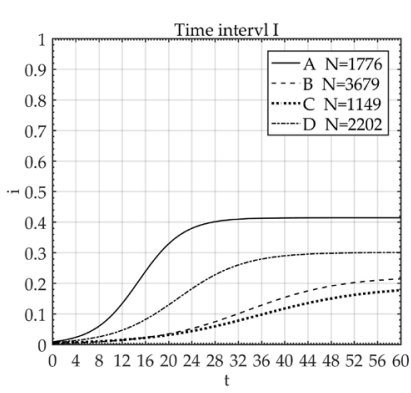

(a)

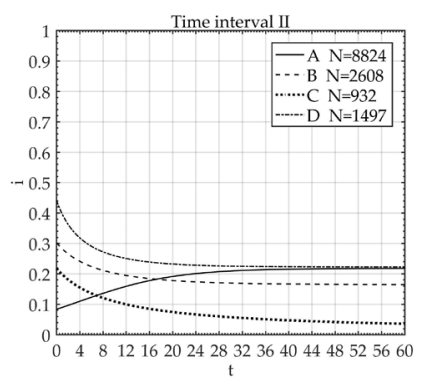

(b)

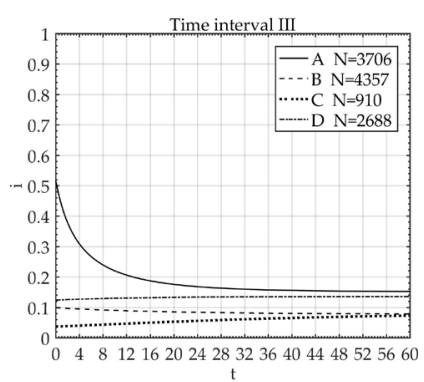

(c)

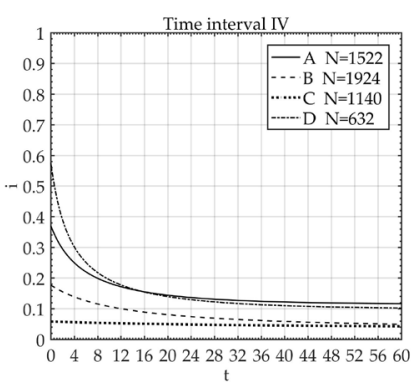

(d) 
lieve that the proportion of initially infected individuals and the probability of information diffusion determine the trend of information diffusion, which is the primary factor affecting new product information diffusion. For the firm-hosted online community, the initial information infected individuals are generally the users who publish new information and the users who make an active response to posts in the first time. If these initially infected individuals are opinion leaders who often publish topic posts to express his/her own opinions to interact with multiple objects and have a high information diffusion probability with a positive community influence. Even if the number is small, it is likely to attract and stimulate a large number of susceptible individuals to contribute new product information in a short time to form a hot spot of interaction, which is conducive to the efficient increase the proportion of infected individuals, to expand the scope of information diffusion in the firm-hosted online community. Therefore, in the field of information diffusion, the influence of opinion leaders cannot be ignored [48]. Williams and Cothrel [60] proposed twelve fundamental lessons to direct how to establish and maintain online communities successfully, after research on the impact of four online communities on business strategy and operations. Our finding has expanded his method of member development and advised community managers should be seeking to attract and cultivate influential users who are considered as "the coordination center of community formation," play their role in information communication bridge, influence, and motivate other users to contribute content by diffusing their knowledge.

At the same time, we found that although the number of user nodes fluctuated within each time interval, the number of infected nodes showed a downward trend. As shown in Figure 4, even if there are a large number of user nodes participating in information diffusion in the community (see the lines with squares), the number of infected nodes is significantly reduced in comparison (see the lines with triangles).

This result indicates that although the sharing and exchange of hot topics of product innovation function attract a large number of users to participate in the information diffusion activities in various time intervals, there are only a few users who actively express new views on new products after they have a preliminary understanding of new product information.

\section{Figure 4}

Comparison between the users participating in the diffusion of new product information and the infected individuals: (a) Topic A; (b) Topic B; (c) Topic C; (d) Topic D

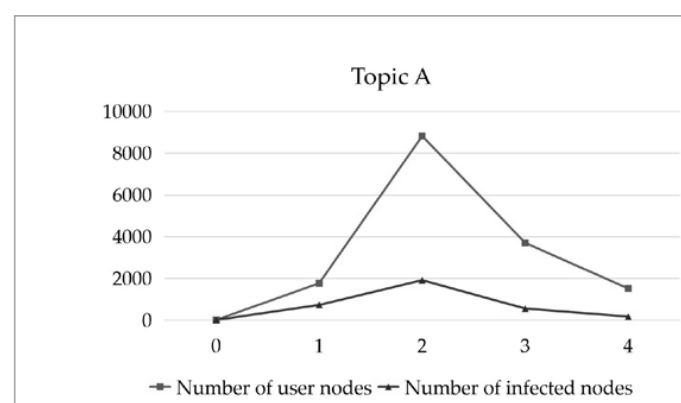

(a)

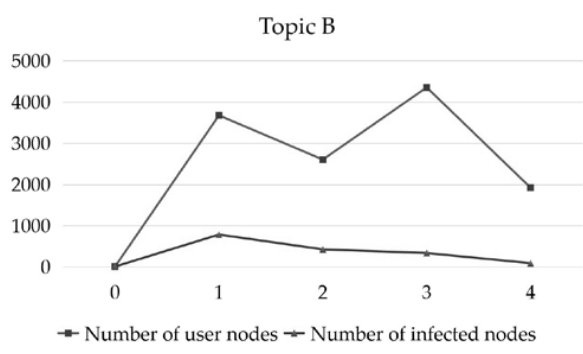

(b)

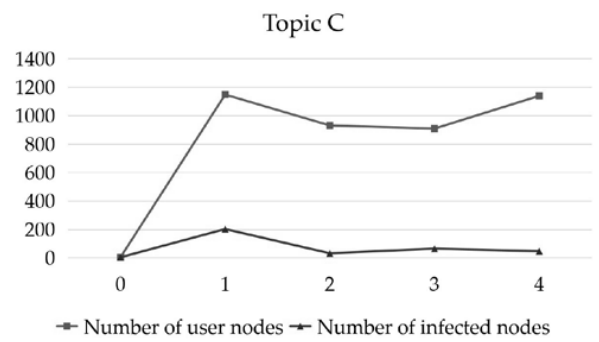

(c)

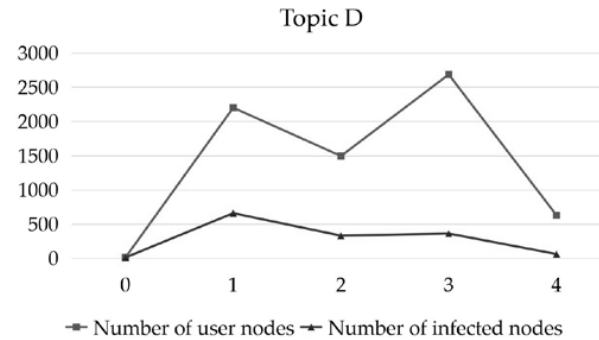

(d) 
Most individuals quickly recover from the infected state. Instead of choosing re-diffusion information, they become the susceptible state again. We suspect that the reason for this result may be closely related to the type of user whose diffusion force is 0 . Therefore, to explain why the number of infected individuals is an apparent disparity compared with the number of participating users as shown in Figure 4, we further use regression analysis to test the causal relationship that causes significant differences in the number of users in different states in the process of information diffusion. Then using the network analysis method, the paper explains the reasons that affect the information diffusion of infected individuals from the perspective of the network structure characteristics of each time interval of the Xiaomi forum.

\section{Regression analysis}

We assume that the probability of information diffusion would be affected by the number of users with diffusion capability of 0 , and also by the number of users with significant influence. Therefore, we take the number of users with diffusion capability of 0 (NNDCU) and the number of significant influence users (NSIU) as independent variables, and the information diffusion probability (IDP) as dependent variables. Because of the vast differences in the study variable data, to make the regression results more robust, the variables were logarithmized. This paper uses SPSS22.0 for data processing analysis and tests the hypothesis, as shown in Table 3-5.

\section{Table 3}

Descriptive statistics and correlations

\begin{tabular}{l|c|c|c|c|c}
\hline \multicolumn{1}{c|}{ Variable } & Mean & Std. dev & 1 & 2 & 3 \\
\hline 1- NNDCU & 3.345 & 0.331 & 1.00 & & \\
\hline 2- NSIU & 1.679 & 0.309 & $0.610^{*}$ & 1.00 & \\
\hline 3- IDP & -0.265 & 0.026 & -0.316 & 0.300 & 1.00 \\
\hline
\end{tabular}

* Correlation is significant at the 0.05 level (2-tailed).

\section{Table 4}

Model results

\begin{tabular}{c|c|c|c|c}
\hline Model & Constant & R & R square & F value \\
\hline 1 & -0.169 & $0.697^{\mathrm{a}}$ & 0.486 & $6.141^{*}$ \\
\hline
\end{tabular}

* Correlation is significant at the 0.05 level (2-tailed). a: dependent variable LN(IDP)
Table 5

Model hypothesis and test results

\begin{tabular}{l|l|c|c}
\hline \multicolumn{2}{|c|}{ Hypothesis } & Beta & Result \\
\hline H1 & $\begin{array}{l}\text { The number of users with } \\
\text { diffusion capability of 0 has a } \\
\text { positive impact on information } \\
\text { diffusion. }\end{array}$ & $-0.793^{* *}$ & Rejected \\
\hline H2 & $\begin{array}{l}\text { The number of significant } \\
\text { influence users has a positive } \\
\text { impact on information } \\
\text { diffusion. }\end{array}$ & $0.784^{* *}$ & Supported \\
\hline
\end{tabular}

** Correlation is significant at the 0.01 level (2-tailed)

From the analysis of the model results, we found that the number of users who are easy to recover to susceptible with diffusion capability of $0(\beta=-0.793$, $\mathrm{P}<0.01$ ) is significantly negatively correlated with the probability of information diffusion, which indicates that the number of users of this type has a significant negative effect on information diffusion, contrary to the H1. The results show that the more the number of users with diffusion capability of 0 , the less the probability of information diffusion. This result also confirms our previous supposition. In contrast, the number of users with significant influence $(\beta=0.784$, $\mathrm{P}<0.01)$ is significantly positively correlated with the probability of information diffusion, indicating that the number of users of this type has a significant positive impact on the information diffusion of each time interval, H2 is supported. Users with significant influence in Xiaomi forum usually show the behavioral characteristics of both active posting and frequent comments, and the posts sent by such users can often attract more users' attention and get comments, which can be usually regarded as opinion leaders in the community and have a more significant influence on other users. Hence, the firm-hosted online community should focus on such highly involved users and take measures to encourage such users to participate in the community activities to widely diffuse new product information. The finding supports prior research that indicates opinion leadership more prominently foster content contribution to the online community hosted by the company, which derived from empirical research by using 855 available data collected by a questionnaire published on consumer-hosted versus company-hosted online communi- 
ties related to sports in Austria [48]. Meanwhile, the crux of the matter is that community managers should pay more attention to those users who are no longer involved in community activities after only participating in one comment, such as the users whose diffusion capability is 0 , which obstructs information diffusion. Previously, Kankanhalli et al. [25] conducted a large-scale survey of knowledge contributors from 10 public organizations in Singapore and established a research model to explain the use of knowledge contributors to the knowledge management system (i.e., EKR). Empirical results indicate that the reluctance of members to contribute is a primary cause of community failure. Our findings confirm once again that the lack of stickiness of community users is the main factor that is expected to result in the instability of online community operation and affect the interests of firms [43]. Therefore, community organizations and managers should consider the incentive mechanism with a reward to encourage users to comment on multiple posts, and actively publish their views on new products, to reduce the number of users with diffusion capability of 0 and improve the efficiency of information diffusion.

\section{Importance analysis of neighbor nodes}

To verify the necessity of considering neighbor nodes in the measurement of influence, we performed a principal component analysis of the elements of user influence that drive information diffusion. Principal component analysis (PCA) is a multivariate statistical method to investigate the correlation between multiple variables, which is used to study how to reveal the internal structure of multiple variables through a few principal components. In the $\mathrm{KMO}$ and Bartlett's test of sphericity, the KMO value is greater than 0.5, and Bartlett's test of sphericity has a P value of less than 0.05, as shown in Table 6. The test results show that there is no correlation between the factors of user influence, and the sample data is suitable for PCA analysis.

Table 6

KMO and Bartlett's test of sphericity

\begin{tabular}{c|c|c|c}
\hline KMO & \multicolumn{3}{|c}{ Bartlett's Test of Sphericity } \\
\hline 0.518 & Approximate Chi-Square & DF & Sig. \\
\hline & 89830.598 & 21 & 0.000 \\
\hline
\end{tabular}

As can be seen from Table 7 , the elements of the user influence of PCA results are roughly divided into three principal components. In Table 8, for each principal component, the analysis items of the principal component are determined by selecting the element variables whose absolute value of the load factor is greater than 0.5 . The three principal components obtained are analyzed as follows. The first principal component is composed of betweenness centrality (0.991) and the clustering coefficient (0.991). It has been shown that the close interaction between user nodes and their neighbors is the key factor affecting the diffusion range of user control information. The second principal component consists of the diffusion capability (0.751) and

Table 7

Total variance explained

\begin{tabular}{c|c|c|c|c|c|c}
\hline \multirow{2}{*}{ Component } & \multicolumn{3}{|c|}{ Initial Eigenvalues } & \multicolumn{3}{c}{ Extraction Sums of Squared Loadings } \\
\cline { 2 - 7 } & Total & \% of Variance & Cumulative \% & Total & \% of Variance & Cumulative \% \\
\hline 1 & 2.092 & 29.890 & 29.890 & 2.092 & 29.890 & 29.890 \\
\hline 2 & 1.385 & 19.779 & 49.669 & 1.385 & 19.779 & 49.669 \\
\hline 3 & 1.033 & 14.761 & 64.430 & 1.033 & & \\
\hline 4 & 0.963 & 13.764 & 78.194 & & & \\
\hline 6 & 0.895 & 12.779 & 90.973 & & & \\
\hline 7 & 0.613 & 8.759 & 99.732 & & & \\
\hline
\end{tabular}


the content similarity (0.711), both of which consider the user influence from the perspective of the user's content interaction with their neighbors. The third principal component is composed of closeness centrality (0.680) and eigenvector centrality (-0.643). They are both used to evaluate the value of users to information diffusion. Moreover, it is further verified that in the information diffusion network, the importance of the user node's influence depends not only on the number of neighbors of the node but also on the influence strength of each neighbor of the node.

Table 8

Component score coefficient matrix

\begin{tabular}{l|c|c|c}
\hline \multirow{2}{*}{} & \multicolumn{3}{|c}{ Component } \\
\cline { 2 - 4 } Degree Centrality & 1 & 2 & 3 \\
\hline Closeness centrality & 0.085 & 0.103 & 0.376 \\
\hline Betweeness centrality & 0.991 & 0.055 & 0.680 \\
\hline Clustering coefficient & 0.991 & 0.050 & 0.050 \\
\hline Eigenvector centrality & 0.118 & 0.470 & -0.643 \\
\hline Diffusion capability & 0.019 & 0.751 & 0.038 \\
\hline Content similarity & 0.075 & 0.711 & 0.355
\end{tabular}

The results derived from this the factor principal component analysis of user influence validate the claims laid in our previous studies that the user's neighbor plays a significant role in information diffusion in the early stage of new product releases. Reviewed on the previous literature, Narang et al. [37] used three publicly available datasets of large social networks, namely, Epinions, Google+, and Livejournal. It took a closeness centrality measure to identify "opinion leaders" who have a significant influence on other nodes in information diffusion. Furthermore, the classical epidemic model is used to evaluate the effect of the interconnection between nodes, which confirms that the interconnection plays a more critical role in the network. Therefore, our findings of an empirical study authenticate the previous research point of view that neighbor nodes with significant influence can better play the advantages of reciprocity. Especially in the firm-hosted online community, to improve the efficiency of information diffusion, users should be encouraged to participate in discussions on multiple different topics. Based on similar interests, they can communicate with users who have a significant influence on some familiar topics and form an interactive connection in the network.
What's more, the community should also use the topics that users are interested in to generate a sense of social identity, and then publish their awareness of new product information, to provide users with opportunities to interact with others.

\section{Network structure analysis}

We further consider the structural characteristics of the information diffusion network of Xiaomi forum and use the modularity [8] measure in the network structure analysis to measure the stability of such large-scale network communities as Xiaomi forum, to find out the reasons that affect the new product information diffusion of infected individuals in Xiao$\mathrm{mi}$ forum. Combined with the inflection points of the number of infected individuals in Figure 4, we choose hot Topic $\mathrm{C}$ as an example to analyze and interpret, as shown in Figure 5.

\section{Figure 5}

Analysis of network structure characteristics of Topic C: (a) Network community numbers and diffusion probability; (b) Community structures in four-time intervals of Topic C

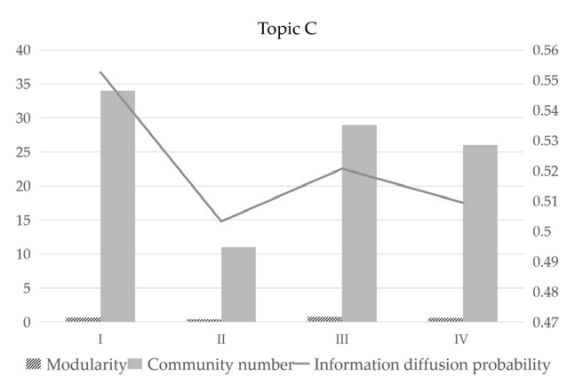

(a)
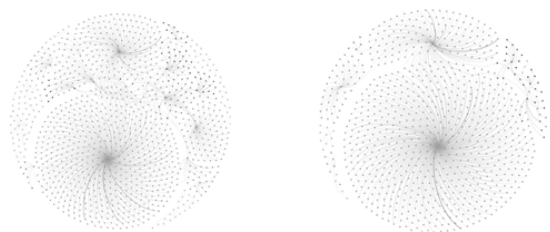

Time interval I

Time interval II

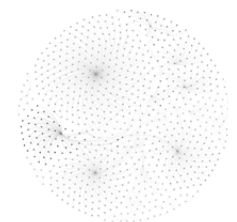

Time interval III

Time interval IV

(b) 
The higher the modularity, the higher the density of links within the community network, and the more sparse and hierarchical obviously of the network community structure. According to Figure 4, we find that the higher the modularity in Figure 4(a), the more the number of network communities, and the higher the probability of information diffusion in this time interval. Figure 4(b) shows a significant difference in the number of communities in the network at various time intervals. This result proves the correctness of our consideration of the clustering coefficient in user influence, and also verifies the diffusion studies of health behaviors in artificially constructed online communities by Centola [6] that in aggregated networks, large-scale diffusion can reach more people and diffuse faster than in random networks. On the contrary, networks with high-level local clustering are inefficient for large-scale diffusion processes. We believe the finding accounts for that in the early stage of information diffusion of new products in communities such as the Xiaomi forum, the high degree of network user aggregation is not propitious to the information diffusion. As shown in time interval II in Figure 4(b), if most users only concentrate in a network community, even if a large number of users participate in it, without evident information inter- action between users (such as mutual comments), users would quickly recover to susceptible, and information cannot effectively diffuse to the outside of the network communities. The result is in agreement with Porter [43] proposed "how to foster and sustain engagement of the virtual community," who took the community of allrecipes.com as an example. It suggests that community managers should encourage users to form multiple small groups based on specific interests, which are helpful to motivate users to contribute content and interaction.

\subsubsection{Forecast for the Arrival Time of New Product Information Diffusion Peak}

Considering the article length, according to the characteristics of the information diffusion of Xiaomi forum, we choose the time interval I when the number of hot topics infected individuals grows to a stable state as an example, and compare and analyze the $i \sim t$ curves and $d i / d t$ curves to illustrate the results.

First of all, in front of the time interval I, the $i \sim t$ curve shows an upward trend, and the number of infected individuals increases significantly, as shown in Figure 6. Moreover, in time interval I, the $d i / d t$ curve shows that when the number of infected individuals in Topic

\section{Figure 6}

Points a, b, c, and d represent the time nodes with the fastest rate of infected individuals of topics A, B, C, and D on $i \sim t$ curves in time interval I
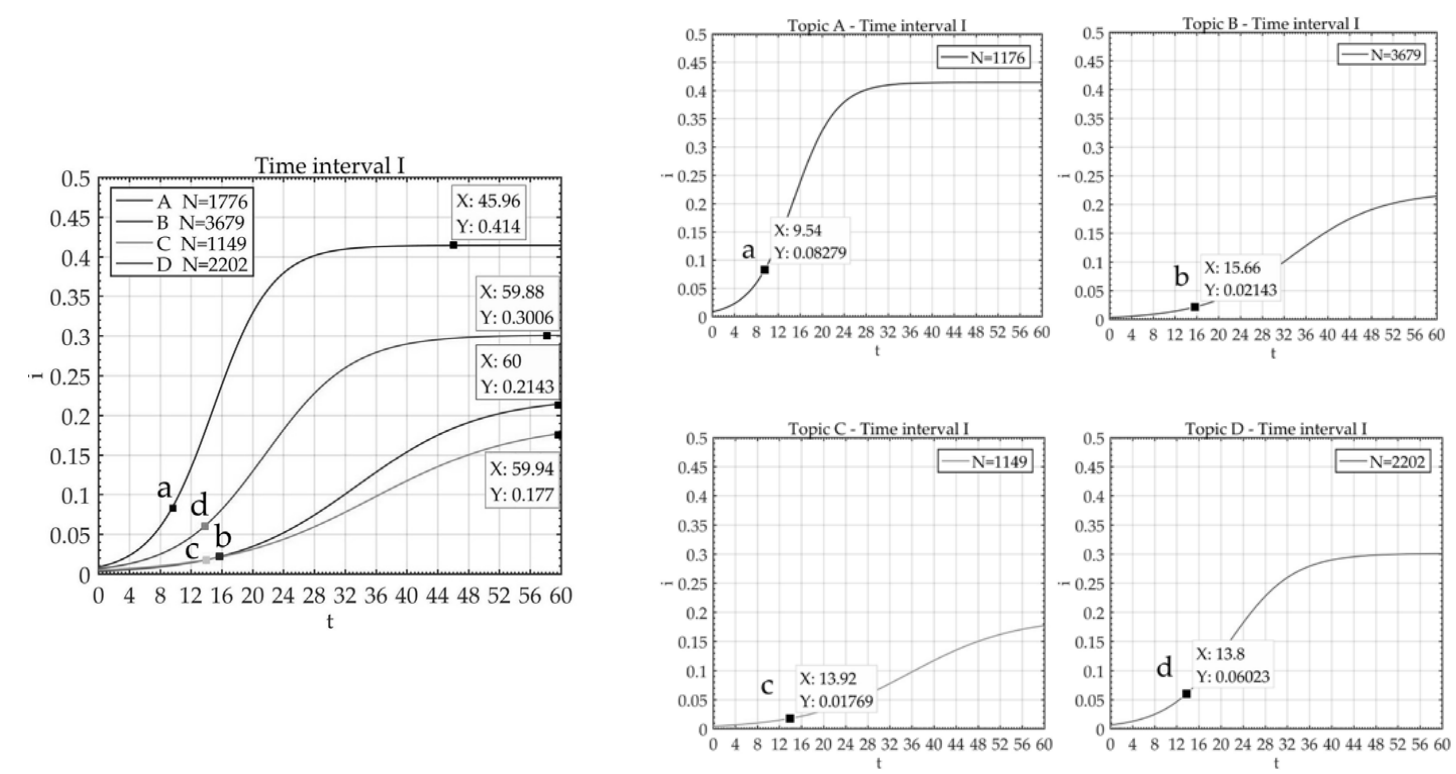
Figure 7

Points with the fastest rate of infected individuals of topics A, B, C, and D on $d i / d t$ curves in time interval I

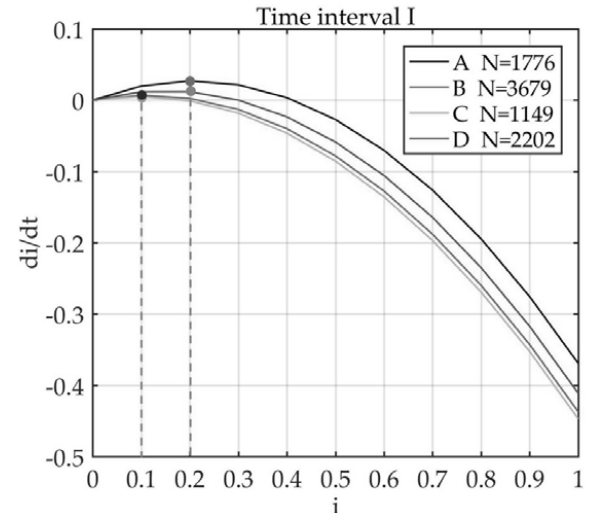

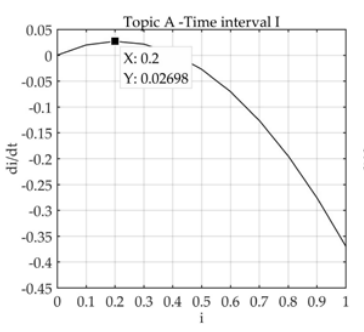
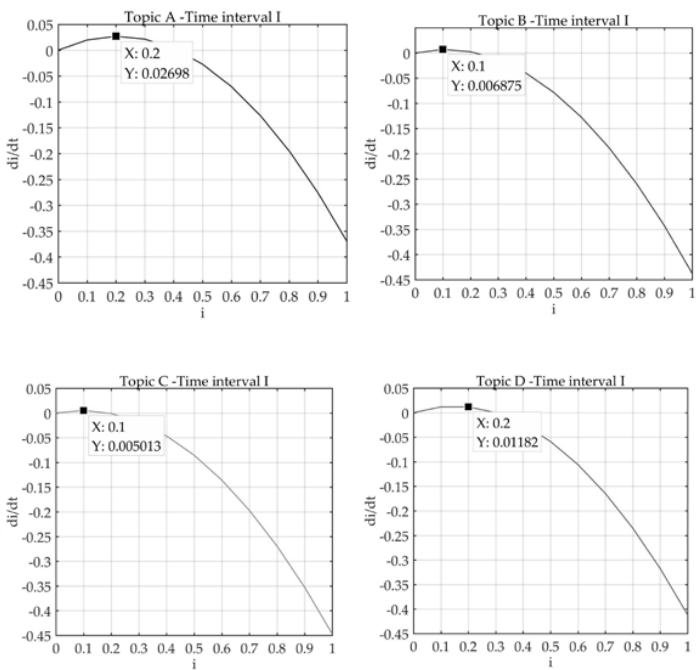

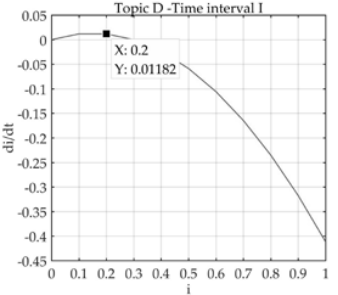

A and Topic D reaches $20 \%$ of the total number of infected individuals, the rate of infected individuals is the fastest. When the number of infected individuals in Topic B and Topic $\mathrm{C}$ reaches $10 \%$ of the total number of infected individuals, the rate of infected individuals is the fastest, as shown in Figure 7. According to the proportion of stable infected individuals, Topic $A$ is 0.414 , Topic B is 0.2143 , Topic $C$ is 0.177 , and Topic $\mathrm{D}$ is 0.3006 , respectively. We marked the key points "a" and "d" of $20 \%$ of the total number of infected in-

\section{Figure 8}

The attention ranking of mobile phones

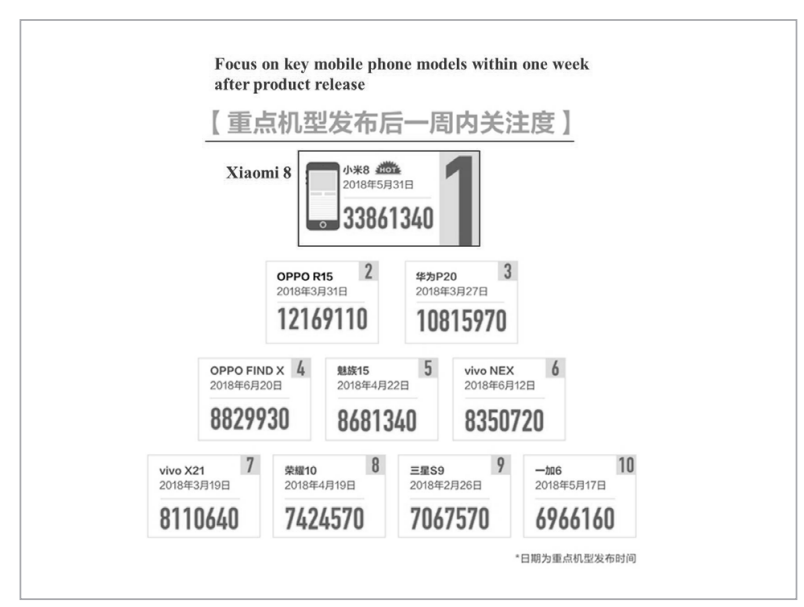

dividuals corresponding to Topic $\mathrm{A}$ and Topic $\mathrm{D}$, and the key points "b" and "c" of $10 \%$ of the total number of infected individuals corresponding to Topic B and Topic $\mathrm{C}$ from the $i \sim t$ curves, which makes us find out the critical time nodes more directly.

We found that in addition to Topic B, the number of infected individuals grew fastest in the range of the other three topics from the 9th time node to the 14th time node. We take 12 hours as a one-time node as described above, so 14-time nodes are 168 hours, which is seven days. We claim that the first seven days after the release of Xiaomi 8/8se mobile phone is the peak of the information diffusion of new products in the Xiaomi forum. According to the white paper on content marketing of the mobile phone industry in 2018 released by information authority (www.toutiao.com) on July 23, 2018, Xiaomi 8 mobile phone ranked first in the attention comparison of key product models one week after the product release [50], as shown in Figure 8. This statistic just verified the results of our model and proved the accuracy of the model in the analysis of the information diffusion process.

In the $i \sim t$ curve of time interval I, we also found that from the 46th time node, the proportion of infected individuals gradually increased to a stable state, as shown in Figure 9. The 46th time node is the 552nd hour in the time interval I, which is the 23rd day since the launch 
Figure 9

Time nodes with a stable number of infected individuals of topics A, B, C, and D on $i \sim t$ curves in time interval I.
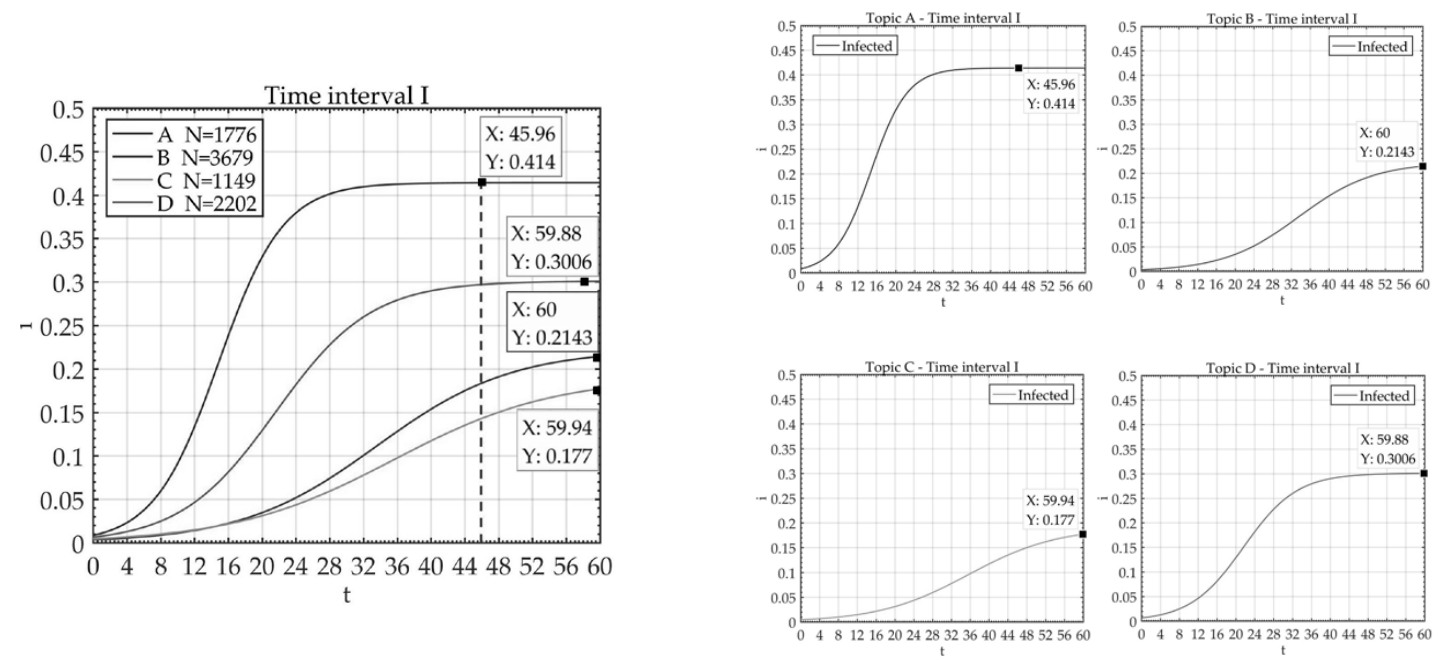

Figure 10

The microblog of Mr. Lei

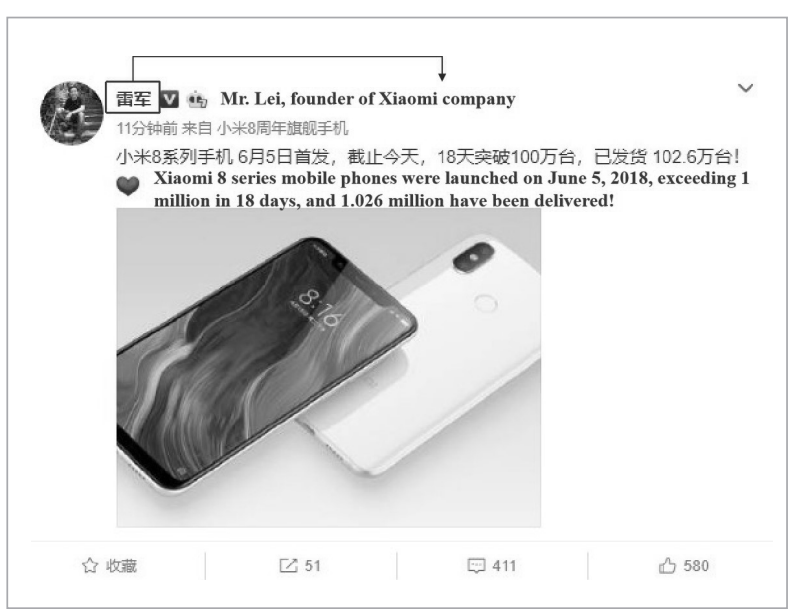

of the Xiaomi 8 mobile phone on May 31, 2018. Mr. Lei, the founder of Xiaomi company, announced on Weibo that Xiaomi 8 series mobile phones had been launched on the official Xiaomi website since June 5, 2018, and had sold more than 1 million in 18 days [27], as shown in Figure 10. By calculating the time nodes, we found that after the 18th day of the first sale of Xiaomi 8 online is converted into a time node, and that is the 46th time node of the curve. This result confirms the reliability of the information diffusion model of the firm-hosted online community to predict the peak value of new product information diffusion once again and also fully embodies the significant application value of the research on a new product information diffusion model to predict product sales.

\section{Discussion}

The objective of this work is to explain the dynamic causes that affect information diffusion during the critical period of early promotion of new products in online communities, through establishing a new product information diffusion model based on user influence in the firm-hosted community. We exploit prior knowledge of the given network and use the grey relation analysis (GRA) to verify the dynamic factors of new product information diffusion explained by the new product information diffusion model and discuss several findings. The research results provide strong support for the effectiveness of the new product information diffusion model.

\subsection{Verification of Dynamic Factors of the New Products Information Diffusion}

Grey relation analysis comes from the model based on the grey theory put forward by Deng [9]. GRA is a mul- 
tiple factor statistical analysis method to determine the correlative grade between factors, which is used to effectively analyze the uncertain correlation between things and system factors or factors, and main behaviors. Generally, based on the sample data of each factor, the GRA is used to describe the strength, size, and order of the relationship between factors. If the trend (direction, size, and speed) of the two factors reflected by the sample data is the same, the correlation between them is large. Inversely, the correlation is low. GRA can provide a quantitative measurement for the development and change of a system, which is suitable for dynamic process analysis, so it has been widely applied in diverse fields of research [18], [47]. Furthermore, because of its simple form, superior calculation efficiency, and high prediction accuracy, GRA can be used for relatively little data and limited information [65]. Therefore, GRA is appropriate for the comparison and verification of the dynamic factors of information diffusion of new products.

In the present work, GRA applied to evaluate the relations between dynamic factors affecting the diffusion of new product information and the diffusion probability of new product information in four hot topics. The current research has identified a set of factors affecting information diffusion. From the perspective of characteristics of the whole network formed by the evolution of four hot topics in four continuous time intervals, including the network modularity (NM) and the number of communities (NC) reflecting the evolution trend of the network community, as well as the eigenvector centrality (EC) reflecting the importance of neighbor nodes. On the other side, from the perspective of individual characteristics of network nodes, including the number of none diffusion capability users (NNDCU) and the number of significant influence users (NUSI). And then, we defined the gray relational coefficient $\xi[k]$, gray relational grade (GRD) $R_{0 i}\left(x_{0}, x_{i}\right)$, and gray relational ordering as follows. The statistical data of four hot topics dimensionless processing were performed for analysis.

The gray relational coefficient $\xi[k]$ represents the relational grade between the reference sequence and the compared sequence at a certain time node. Let $x=$ $\left\{x_{i} \mid i \in \mathrm{I}\right\}$ be a space sequence where $i=(1,2,3, \ldots, m) . x_{i}$ is a factor of the system, and its value at the $k$ th entity in the sequence is $x_{i}[k]$, where $k=(1,2,3, \ldots, n)$. If we denote the reference sequence $x_{0}$ by $x_{0}(1), x_{0}(2), \ldots, x_{0}$ (n), i.e., the information diffusion probability of new products during four continuous time intervals, and the compared sequence $x_{i}$ by $x_{i}(1), x_{i}(2), \ldots, x_{i}(n), x_{i}$, i.e., the main dynamic factors influencing information diffusion of new products. The gray relational coefficient between $x_{0}$ and $x_{i}$ at the $k$ th entity is given by:

$\xi_{i}(k)=\frac{\min _{i} \min _{k}\left|x_{0}[k]-x_{i}[k]\right|+\rho \max _{i} \max _{k}\left|x_{0}[k]-x_{i}[k]\right|}{\left|x_{0}[k]-x_{i}[k]\right|+\rho \max _{i} \max _{k}\left|x_{0}[k]-x_{i}[k]\right|}$,

where $\rho$ is the resolution coefficient, $0<\rho<1$. If $\rho$ is small, and the resolution ability is strong - generally, $\rho=0.5$.

The GRD reflects the degree of correlation between the reference sequence and each compared sequence and describes the trend of correlation between the two sequences. $R_{0 i}\left(x_{0}, x_{i}\right)$ is expressed as:

$$
R_{0 i}\left(x_{0}, X_{i}\right)=\frac{1}{n} \sum_{k=1}^{n} \xi_{i}(k)
$$

where $i=(1,2,3, \ldots, m)$ is the factor, $k=(1,2,3, \ldots, n)$ is the time node, $x_{0}[k]$ is the reference sequence, $x_{i}[k]$ is the compared sequence, $\left|x_{0}[k]-x_{i}[k]\right|$ denotes the absolute difference between the two sequences, and $\min _{i} \min _{k}\left|x_{0}[k]-x_{i}[k]\right|$ and $\max _{i} \max _{k}\left|x_{0}[k]-x_{i}[k]\right|$ are the minimum and maximum of the absolute differences in all compared sequences, respectively.

The primary purpose of GRA is to find the importance order of the influencing factors according to the values of $R_{0 i}\left(x_{0}, x_{i}\right)$ and finally to determine the crucial factors affecting information diffusion of new products. Therefore, the gray relational ordering is a significant index to be compared to the importance of factors. If $R_{0 i}>R_{0 j}$, then the influence of $x_{i}$ on $x_{0}$ is more dominant than that of $x_{j}$ on $x_{0}$. If $R_{0 i}=R_{0 j}$, then the influence of $x_{i}$ on $x_{0}$ is the same as that of $x_{j}$ on $x_{0}$.

\subsection{Key Dynamic Factors on Information Diffusion of New Products}

We use different patterns to represent and distinguish the factors, as shown in Figure 11. NNDCU, NSIU, NM, NC, and EC are related to the information diffusion probability (IDP) of new products in the four hot topics of the firm-hosted online community, respectively. In particular, NNDCU has a clear correlation with IDP in Topic A, and Topic C. NSIU is significantly related to IDP of Topic B. Moreover, NM 
Figure 11

Gray relation analysis results of topics A, B, C, and D: (a) Topic A; (b) Topic B; (c) Topic C; (d) Topic D

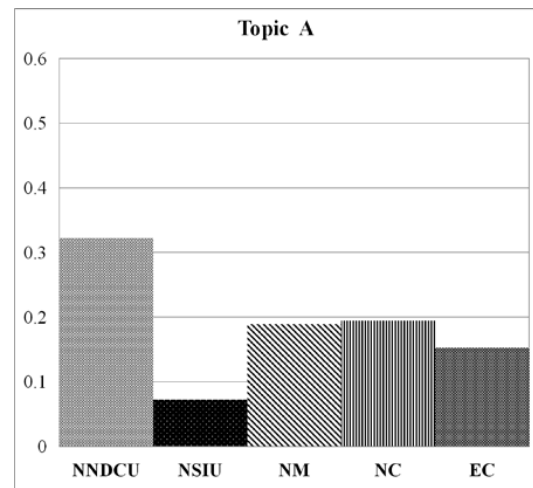

(a)

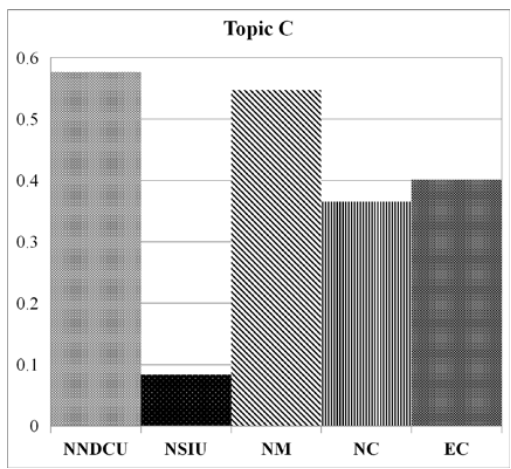

(c)

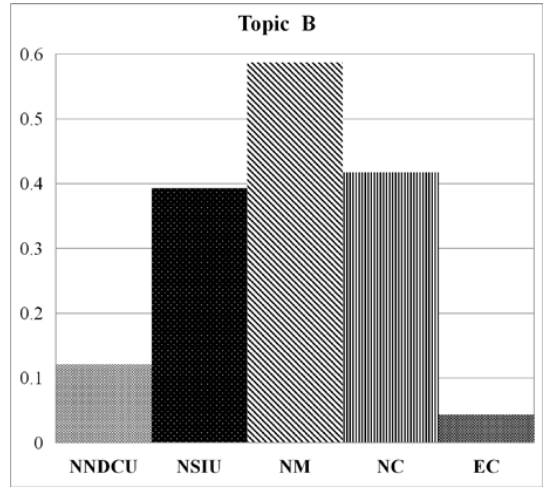

(b)

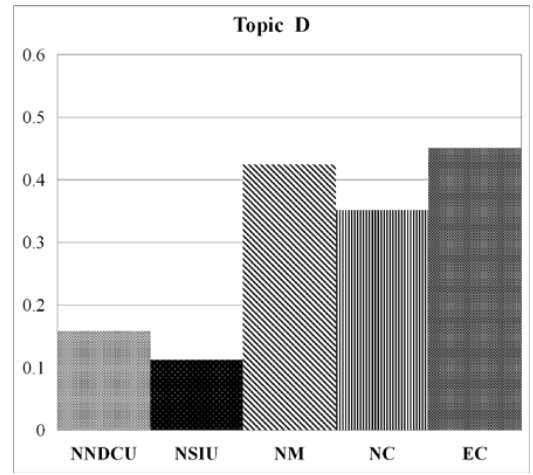

(d) and NC show a high correlation with IDP in Topics B, C, and D. EC also has apparent relativity with IDP in Topic C and Topic D.

In general, the results of GRA have verified the validity of the dynamic factors that affect the information diffusion of new products explained by the new product information diffusion model of the firm-hosted community.

We further rank the importance of the relational grade of the influencing factors, to more directly represent the influencing factors that play a critical role in the information diffusion of new products in the four hot topics, as shown in Table 9. It is worth noting that the meaningful findings have practical application value for thinking and putting forward corresponding community management strategies to promote the vast and rapid diffusion of new product information.

\section{Table 9}

The ranking of the relational grades of influence factors of topics A, B, C, and D

\begin{tabular}{l|c|c|c|c|c}
\hline & \multicolumn{5}{|c}{ Gray relational ordering } \\
\cline { 2 - 6 } & 1 & 2 & 3 & 4 & 5 \\
\hline Topic A & NNDCU & NC & NM & EC & NSUI \\
\hline Topic B & NM & NC & NSUI & NNDCU & EC \\
\hline Topic C & NNDCU & NM & EC & NC & NSUI \\
\hline Topic D & EC & NM & NC & NNDCU & NSUI \\
\hline
\end{tabular}

First of all, by comparing the importance of the influencing factors in the four topics, we find that NM and NC rank higher in importance. The result has shown that the key factors affecting the information diffusion effect of the new product are the network 
modularity and the number of communities in the firm-hosted online community. As the results we have obtained in the preceding sections, the more the value of modularity of community network, the stronger the community structure [39]. The results suggest that the formation of multiple small communities in the firm-hosted online community is more conducive to users' contributions and sharing of new product information on specific topics. Therefore, how to form more network communities, reduce the largescale aggregation of users, and promote the rapid and extensive interaction and diffusion of information among users in multiple communities is the primary consideration in the research of new product information diffusion in firm-hosted online communities.

Secondly, with different topics of product innovation function, the importance of the key factors affecting the information diffusion of new products has also changed. Specifically, NNDCU is the key factor influencing the information diffusion of new products in Topic A of "Snapdragon 845" and Topic C of "Transparent exploration version of the under-screen fingerprint." It provides crucial suggestions for community managers on how to reduce the impact of new product information diffusion. Community managers should be commit to taking necessary measures to reduce the proliferation of users (i.e., those who have none diffusion capability) who only make one comment. To attract and encourage more users to share and contribute their cognition and understanding about new products and promote the effective information diffusion of new products.

Thirdly, unexpectedly, the influence of NSIU on the other three topics seems not significant, in addition to being an important factor in the information diffusion of new products in Topic B of "Infrared face recognition unlocking." That is different from the previous studies pointed out the significant influence users (i.e., Opinion leaders) are the remarkable influencing factors of information interaction activities of community members. In particular, we further found that the EC is a key factor that cannot ignore in Topic $\mathrm{C}$ of "Transparent exploration version of the under-screen fingerprint" and Topic D of "The world's first dual-frequency GPS of the world." That also demonstrates the importance of network neighborhood in the information diffusion of new products. Based on the above result, because EC takes into consideration the extent to the influence of neighbor nodes in the network relative to other nodes connected to them [3]. Hence, making community users to establish a connection with more influential network neighbors in interactive activities plays a more critical role in the information diffusion of new products, rather than the number of significant influence users in the community are more conducive to information diffusion. The interesting new result we found deserves the attention of community managers. Meanwhile, the findings also put forward the future research direction for how to establish and enhance the interaction between general users and opinion leaders with significant influence to promote the diffusion of new product information.

\section{Conclusions and Limitations}

This study established the new product information diffusion model of firm-hosted online communities by using the epidemic model SIS. This paper discussed and explained the dynamic information diffusion process and its dynamic causes among users during the critical period of early promotion of new products in online communities. As very little research on the driving force of information diffusion in the early stage of new products entering the market, we attempted to explore this field from the perspective of information diffusion probability that affects the change of users' state in the firm's community. As well as explain the driving force of information diffusion and provide a theoretical and practical basis for firms to put forward effective management and marketing strategies of new products. We filled in the blank of the research on the mechanism of new product information diffusion in the firm-hosted online community. We supplemented the research on the antecedent of user-generated effective information mining, to provide the research basis for analyzing the valuable content generated by users.

We used the individual influence of users as the driving force to affect the state transfer of the community users and quantified the probability of information diffusion. The results revealed that the probability of information diffusion significantly affects the number of infected individuals and the rate of infected. We further found that the proportion of initially infect- 
ed individuals is a crucial factor in determining the trend of information diffusion. Community managers should fully exploit the initially infected individuals with sufficient influence, and encourage such users to actively post, maintain the discussion enthusiasm of new product innovation function topics, and attract the active interaction of susceptible individuals. The infected individuals can effectively control the trend of product public opinion, and help firms establish the reputation of new product functions. Whether it is the positive word-of-mouth recognized by users, or contrary word-of-mouth about product defects, that contribute to product iterative innovation, it has a vital role in promoting online marketing products. Moreover, those users whose diffusion capability is 0 are the influencing factors that significantly hinder information diffusion. Community practitioners should take measures to reduce the probability of users with the diffusion capability of 0 becoming zombie users. After this kind of users return to susceptible, new product information similar to the information of interest in their historical data is pushed to them, it would be better to attract such users to participate in the diffusion of new product information.

It should not be ignored that both in number and quality of users' neighbors play an essential role in the process of information diffusion. In the large-scale firm community networks, the more network communities formed, and the smaller the size of neighborhood accounts for the total number of community users, the more conducive to the diffusion of new product information. Inversely, the high-level clustering coefficient of the network hinders the large-scale information diffusion. That is contrary to the information diffusion of highly clustered social networks. Hence, community managers must control the number of neighborhood nodes while paying attention to the quality of users' neighborhood nodes, to form more network clusters in the community, strengthen the information interaction between network clusters, and improve the efficiency of information diffusion of new products.

Finally, we used the actual mobile phone online sales data of Xiaomi Company to test the accuracy and validity of the information diffusion model in predicting the peak time of new product information diffusion in the firm-hosted online community. Our research results enrich the literature on the mechanism of infor- mation diffusion in firm-hosted online communities, increase our understanding of information diffusion methods, and influence modes based on user interaction behavior. In parallel, help community practitioners to design firm-hosted online community systems more effectively and make new product development and marketing decisions in time.

The purpose is to verify the effectiveness of the results of the new product information diffusion model, and we first compared the relevant researches of multiple online communities based on open datasets. We affirmed that our research results are consistent with the previous, which shows that our research method is reliable. Moreover, we utilize the grey relational analysis to verify the validity of the factors we proposed. Because the neighborhood nodes with significant influence can establish the relationship between individuals of different network communities, better evoke the community users' general sense of shared identity, and create more opportunities for the relationship between community individuals. Therefore, from the perspective of information diffusion of new products, it is more important to consider how to establish a neighborhood relationship with significant influence nodes than there are more significant influence nodes in the community. That is the meaningful results we found. It also reconfirms the importance of neighborhood nodes in the firm-hosted online community once more.

Several limitations restrict this study. We used the official forum of Xiaomi as a typical example of firm-hosted online communities to test our research model. Although Xiaomi forum is one of the most visited online communities in China, our research results may not be directly applicable to other communities. That is due to differences in the characteristics of the community and the goals to be achieved. Users of the Xiaomi forum purposefully participate in the activities of the firm-hosted online community. They obtain information about products and services and determine whether to purchase new products through the exchange of experience, rather than just extending the social circle to get the information they are interested in as in other social networks. Additionally, the pattern of information diffusion may also be affected by the popularity of information topics related to new products. In particular, users' emotional inclination towards product brands and competing 
products also have an impact on the information diffusion of new products. In the future, we will conduct confirmatory research in many similar firm communities to make the results more convincing.

\section{Funding}

This research was funded by the National Key R\&D Program of China under Grant No. 2018YFB1700800

\section{References}

1. Abrahams, A. S., Jiao, J., Fan, W. G., Wang, G. A., Zhang, Z. J. What's Buzzing in the Blizzard of Buzz? Automotive Component Isolation in Social Media Postings. Decision Support Systems, 2013, 55(4), 871-882. https:// doi.org/10.1016/j.dss.2012.12.023

2. Barrat, A., Barthélemy, M., Vespignani, A. Dynamical Processes on Complex Networks. Cambridge University Press, Cambridge, 2008. https://doi.org/10.1017/ CBO9780511791383

3. Battaglio R. P., Hall, J. L. Exploring the Frontiers of Administrative Behavior. Public Administration Review, 201, 80(1), 6-8. https://doi.org/10.1111/puar.13148

4. Cannarella, J., Spechler, J. A. Epidemiological Modeling of Online Social Network Dynamics. ArXiv, 2014. https://arxiv.org/abs/1401.4208.

5. Carrera, B., Jung, J. Y. SentiFlow: An Information Diffusion Process Discovery Based on Topic and Sentiment from Online Social Networks. Sustainability, 2018, 10(8), 2731. https://doi.org/10.3390/su10082731

6. Centola, D. The Spread of Behavior in an Online Social Network Experiment. Science, 2010, 329(5996), 11941197. https://doi.org/10.1126/science.1185231

7. Cho, V., Chan A. A Study on the Influence of EWOM Using Content Analysis: How Do Comments on Value for Money, Product Sophistication and Experiential Feeling Affect Our Choices? Enterprise Information Systems, 2017, 11(6), 927-948. https://doi.org/10.1080/175 17575.2016 .1154610

8. Clauset, A., Newman, M. E. J., Moore, C. Finding Community Structure in Very Large Networks. Physical Review E, 2004, 70(6), 066111. https://doi.org/10.1103/ PhysRevE.70.066111

9. Deng, J. L. Control Problems of Grey Systems. Systems \& Control Letters, 1982, 1(5), 288-294.https://doi. org/10.1016/S0167-6911(82)80025-X and the State Key Program of National Social Science Foundation of China under Grant No. 16AZD004.

\section{Acknowledgments}

The authors would like to convey their appreciation and gratitude to the anonymous reviewers. Their remarks and suggestions were extremely insightful and enabled us to improve the quality of the manuscript.

10. Divakaran, P. K. P. Pre-Release Member Participation as Potential Predictors of Post-Release Community Members' Adoption Behaviour: Evidence from the Motion Picture Industry. Behaviour \& Information Technology, 2013, 32(6), 545-559.https://doi.org/10.1080/01 44929X.2012.681069

11. Du, Y. J., Peng, B., Su, F. H., Cheng, F., Du, S. Y. Mining Hot-Personae Approach Based on Local Social Microblog Graph. Information Technology and Control, 2019, 48(4), 522-537. https://doi.org/10.5755/j01. itc.48.4.21950

12. Feng, L., Hu, Y. Q., Li, B. W., Stanley, H. E., Havlin, S., Braunstein, L. A. Competing for Attention in Social Media under Information Overload Conditions. PLoS ONE, 2015, 10(7), e0126090. https://doi.org/10.1371/ journal.pone.0126090

13. Gartner Research. China Summary Translation: 'SWOT: Xiaomi, Smart Devices, Worldwide'. https:// www.gartner.com/en/documents/3892005. Accessed on October 26, 2018.

14. Gartner. Gartner Says Demand for Top Chinese Brands Drove Worldwide Smartphone Sales in Third Quarter 2018. https://www.gartner.com/en/newsroom/pressreleases/2018-12-03-gartner-says-demand-for-top-chinese-brands-drove-worl. Accessed on December 3, 2018.

15. Gartner. Gartner Says Global Smartphone Sales Stalled in the Fourth Quarter of 2018. https://www.gartner. com/en/newsroom/press-releases/2019-02-21-gartner-says-global-smartphone-sales-stalled-in-thefourth-quart. Accessed on February 21, 2019.

16. Gartner. Gartner Says Worldwide Sales of Smartphones Returned to Growth in First Quarter of 2018. https:// www.gartner.com/en/newsroom/press-releases/201805-29-gartner-says-worldwide-sales-of-smartphonesreturned-to-growth-in-first-quarter-of-2018. Accessed on May 29, 2018. 
17. Granovetter, M. S. The Strength of Weak Ties. American Journal of Sociology, 1973, 78, 1360-1380.

18. Guo, J., Kang, Y. Characterization of Sulfate-Reducing Bacteria Anaerobic Granular Sludge and Granulometric Analysis with Grey Relation. Korean Journal of Chemical Engineering, 2018, 35(9), 1829-1835. https:// doi.org/10.1007/s11814-018-0092-y

19. Guo, W., Liang, R. Y., Wang, L., Peng, W. Exploring Sustained Participation in Firm-Hosted Communities in China: The Effects of Social Capital and Active Degree. Behaviour \& Information Technology, 2017, 36(3), 223242. https://doi.org/10.1080/0144929X.2016.1212402

20. Guo, W., Zheng, Q., An, W. J., Peng, W. User Roles and Contributions During the New Product Development Process in Collaborative Innovation Communities. Applied Ergonomics, 2017, 63, 106-114. https://doi. org/10.1016/j.apergo.2017.04.013

21. Han, X. T., Niu, L. On Charactering of Information Propagation in Online Social Networks. Journal of Networks, 2013, 8(1), 124-132. https://doi.org/10.4304/ jnw.8.1.124-131

22. Hill, S., Provost, F., Volinsky, C. Network-Based Marketing: Identifying Likely Adopters via Consumer Networks. Statistical Science, 2006, 21(2), 256-276. https:// doi.org/10.1214/088342306000000222

23. Ienco, D., Bonchi, F., Castillo, C. The Meme Ranking Problem: Maximizing Microblogging Virality. 2010 IEEE International Conference on Data Mining Workshops, (ICDMW 2010), Sydney, NSW, December 13, 2010, 328-335. https://doi.org/10.1109/ICDMW.2010.127

24. Jalili, M., Perc, M. Information Cascades in Complex Networks. Journal of Complex Networks, 2017, 5(5), 665-693. https://doi.org/10.1093/comnet/cnx019

25. Kankanhalli, A., Tan, B. C. Y., Wei, K. K. Contributing Knowledge to Electronic Knowledge Repositories: An Empirical Investigation. MIS Quarterly, 2005, 29(1), 113-143. https://doi.org/10.2307/25148670

26. Kitsak, M., Gallos, L. K., Havlin, S., Liljeros, F., Muchnik, L., Stanley, H. E., Makse, H. A. Identification of Influential Spreaders in Complex Networks. Nature Physics, 2010, 6, 888-893. https://doi.org/10.1038/nphys1746

27. Lei, J. The Sales Volume of the Xiaomi 8 Series Mobile Phones Exceeded 1 Million Units in 18 Days and Have Been Delivered 1.026 Million Units. http://news. mydrivers.com/1/581/581921.html. Accessed on June 23, 2018.
28. Li, M. W., Jia, S. L., Du, W. Y. Fans as a Source of Extended Innovation Capabilities: A Case Study of Xiaomi Technology. International Journal of Information Management, 2019, 44, 204-208. https://doi.org/10.1016/j. ijinfomgt.2018.09.007

29. Li, M., Wang, X., Gao, K., Zhang, S. S. A Survey on Information Diffusion in Online Social Networks: Models and Methods. Information, 2017, 8(4), 118. https://doi. org/10.3390/info8040118

30. Liang, R. Y., Guo, W., Yang, D. Q. Mining Product Problems from Online Feedback of Chinese Users. Kybernetes, 2017, 46(3), 572-586. https://doi. org/10.1108/K-03-2016-0048

31. Liang, R. Y., Guo, W., Zhang, L. H. Exploring Oppositional Loyalty and Satisfaction in Firm-Hosted Communities in China: Effects of Social Capital and E-Quality. Internet Research, 2019, Vol. ahead-of-print. https:// doi.org/10.1108/INTR-07-2018-0344

32. Liu, C., Zhang, Z. K. Information Spreading on Dynamic Social Networks. Communications in Nonlinear Science and Numerical Simulation, 2014, 19(4), 896-904. https://doi.org/10.1016/j.cnsns.2013.08.028

33. Lü, L. Y., Zhou, T., Zhang, Q. M., Stanley, H. E. The H-Index of a Network Node and its Relation to Degree and Coreness. Nature Communications, 2016, 7, 10168. https://doi.org/10.1038/ncomms10168

34. Mahajan, V., Muller, E., Bass, F. M. New Product Diffusion Models in Marketing: A Review and Directions for Research. Journal of Marketing, 1990, 54(1), 1-26. https://doi.org/10.1177/002224299005400101

35. Mao, J. X., Liu, Y. Q., Zhang, M., Ma, S. P. Social Influence Analysis for Micro-Blog User Based on User Behavior. Chinese Journal of Computers, 2014, 37(4), 791800. https://doi.org/10.3724/SP.J.1016.2014.00791

36. Na, Y. K., Kang, S. Sustainable Diffusion of Fashion Information on Mobile Friends-Based Social Network Service. Sustainability, 2018, 10(5), 1474. https://doi. org/10.3390/su10051474

37. Narang, R., Sarin, S., Singh, P., Goyal, R. Impact of Reciprocity in Information Spreading Using Epidemic Model Variants. Information, 2018, 9(6), 136. https:// doi.org/10.3390/info9060136

38. Newman, M. E. J. The Structure and Function of Complex Networks. SIAM Review, 2003, 45(2), 167-256. https://doi.org/10.1137/S003614450342480

39. Newman, M. E. J., Girvan, M. Finding and Evaluating Community Structure in Networks. Physical Review 
E, 2004, 69(2), 026113. https://doi.org/10.1103/PhysRevE.69.026113

40. Obregon, J., Song, M., Jung, J. Y. InfoFlow: Mining Information Flow Based on User Community in Social Networking Services. IEEE Access, 2019, 7, 4802448036. https://doi.org/10.1109/ACCESS.2019.2906081

41. Pastor-Satorras, R., Castellano, C., Van Mieghem, P., Vespignani, A. Epidemic Processes in Complex Network. Reviews of Modern Physics, 2015, 877(3), 925-979. https://doi.org/10.1103/RevModPhys.87.925

42. Porter, C. E., Donthu, N. Cultivating Trust and Harvesting Value in Virtual Communities. Management Science, 2008, 54(1), 113-128. https://doi.org/10.1287/ mnsc.1070.0765

43. Porter, C. E., Donthu, N., MacElroy, W. H., Wydra D. How to Foster and Sustain Engagement in Virtual Communities. California Management Review, 2011, 53(4): 80110. https://doi.org/10.1525/cmr.2011.53.4.80

44. Rogers, E. M. Diffusion of Innovations. NY Free Press, New York, 1995.

45. Sheikhahmadi, A., Nematbakhsh, M. A., Zareie, A. Identification of Influential Users by Neighbors in Online Social Networks. Physica A: Statistical Mechanics and its Applications, 2017, 486, 517-534. https://doi. org/10.1016/j.physa.2017.05.098

46. Shih, C. C., Lin, T. M. Y., Luarn, P. Fan-Centric Social Media: The Xiaomi Phenomenon in China. Business Horizons, 2014, 57(3), 349-358. https://doi.org/10.1016/j. bushor.2013.12.006

47. Sun, G. D., Guan, X., Yi, X., Zhou, Z. Grey Relational Analysis Between Hesitant Fuzzy Sets with Applications to Pattern Recognition. Expert Systems with Applications, 2018, 92, 521-532. https://doi.org/10.1016/j. eswa.2017.09.048

48. Teichmann, K., Stokburger-Sauer, N. E., Plank, A., Strobl, A. Motivational Drivers of Content Contribution to Company-Versus Consumer-Hosted Online Communities. Psychology \& Marketing, 2015, 32(3), 341-355. https://doi.org/10.1002/mar.20783

49. Torche, F., Valenzuela, E. Trust and Reciprocity: A Theoretical Distinction of the Sources of Social Capital. European Journal of Social Theory, 2011, 14(2), 181-198. https://doi.org/10.117r/1368431011403461

50. Toutiao. Toutiao Released a White Paper on Content Marketing in the Mobile Industry to Interpreting the First Half of the 2018 Marketing War. https://www.tou- tiao.com/a6581338327968907779/. Accessed on July 23, 2018 .

51. Ullah, F., Lee, S. C. Identification of Influential Nodes Based on Temporal-Aware Modeling of Multi-Hop Neighbor Interactions for Influence Spread Maximization. Physica A: Statistical Mechanics and its Applications, 2017, 486, 968-985. https://doi.org/10.1016/j. physa.2017.05.089

52. von Hippel, E. User as innovators. Technology Review, 1978, 80(3), 31-39. https://doi.org/10.2307/2598803

53. Wang, C., Yang, X. Y., Xu, K., Ma, J. F. Seir-Based Model for the Information Spreading over SNS. Acta Electronica Sinica, 2014, (11), 2325-2330. https://doi. org/10.3969/j.issn.0372-2112.2014.11.031

54. Wang, Q. Y., Lin, Z., Jin, Y. H., Cheng, S. D., Yang, T. ESIS: Emotion-Based Spreader-Ignorant-Stifler Model for Information Diffusion. Knowledge-Based Systems, 2015, 81, 46-55. https://doi.org/10.1016/j.knosys.2015.02.006

55. Wang, Z. G., Wang (Avery. W), Y., Wu, S. B. The Activity Evaluation Model and Sustainable Interactive Management Strategies of Online User Innovation Community. Sustainability, 2018, 10(7), 2113. https://doi. org/10.3390/su10072113

56. Wasserman, S., Faust, K. Social Network Analysis. Cambridge University Press, Cambridge, 1994. https:// doi.org/10.1017/CBO9780511815478

57. Watts, D. J. Six Degrees: The Science of a Connected Age. W. W. Norton, New York, 2003.

58. Watts, D. J. Small Worlds: The Dynamics of Networks between Order and Randomness. Princeton University Press, Princeton, 1999. https://doi. org/10.1515/9780691188331

59. Watts, D. J., Strogatz, S. H. Collective Dynamics of 'Small World' Networks. Nature, 1998, 393, 440-442. https://doi.org/10.1038/30918

60. Williams, R. L., Cothrel, J. Four Smart Ways to Run Online Communities. Sloan Management Review, 2000, 41(4), 81-91.

61. Xiao, Y. P., Li, S. Y., Liu, Y. B. An Information Diffusion Dynamic Model Based on Social Influence and MeanField Theory. Acta Physica Sinica, 2017, 66(3), 24-37. https://doi.org/10.7498/aps.66.030501

62. Xiong, F., Liu, Y., Zhang, Z. J., Zhu, J., Zhang, Y. An Information Diffusion Model Based on Retweeting Mechanism for Online Social Media. Physics Letters A, 2012, 
376(30-31), 2103-2108. https://doi.org/10.1016/j.physleta.2012.05.021

63. Xu, D. L., Pan, J. C., Wang, B. L., Liu, M., Kang, Q. M. Information Dissemination Model of Microblogging with Internet Marketers. Journal of Information Processing Systems, 2019, 15(4), 853-864. https://doi.org/10.3745/ JIPS.04.0126

64. Xu, R., Li, H., Xing, C. Research on Information Dissemination Model for Social Networking Services. International Journal of Computer Science Applied, 2013, 2(1), 1-6.

65. Zeng, B., Duan, H. M., Zhou, Y. F. A New Multivariable Grey Prediction Model with Structure Compatibility.
Applied Mathematical Modelling, 2019, 75, 385-397. https://doi.org/10.1016/j.apm.2019.05.044

66. Zheng, Q., Guo, W., An, W. J., Wang, L., Liang, R. Y. Factors Facilitating User Projects Success in Co-Innovation Communities. Kybernetes, 2018, 47(4), 656-671. https://doi.org/10.1108/K-01-2017-0012

67. Zhou, S. Z. Xiaomi's Active Users Have Reached Hundreds of Millions of People Around the World. http:// www.egsea.com//news/detail?id=448347. Accessed on July 03, 2019.

68. Zhu, Y. X., Zhang, X. G., Sun, G. Q., Tang, M., Zhou, T., Zhang, Z. K. Influence of Reciprocal Links in Social Networks. PLoS ONE, 2014, 9(7), e103007. https://doi. org/10.1371/journal.pone.010300r7 